\title{
An anisotropic eigenvalue problem of Stekloff type and weighted Wulff inequalities
}

\author{
Lorenzo Brasco and Giovanni Franzina
}

\begin{abstract}
We study the Stekloff eigenvalue problem for the so-called pseudo $p$-Laplacian operator. After proving the existence of an unbounded sequence of eigenvalues, we focus on the first nontrivial eigenvalue $\sigma_{2, p}$, providing various equivalent characterizations for it. We also prove an upper bound for $\sigma_{2, p}$ in terms of geometric quantities. The latter can be seen as the nonlinear analogue of the Brock-Weinstock inequality for the first nontrivial Stekloff eigenvalue of the (standard) Laplacian. Such an estimate is obtained by exploiting a family of sharp weighted Wulff inequalities, which are here derived and appear to be interesting in themselves.
\end{abstract}

Mathematics Subject Classification (2010). 35P30, 47A75, 34B15.

Keywords. Stekloff eigenvalue problem, Pseudo $p$-Laplacian, Wulff inequality.

\section{Introduction}

\subsection{The problem}

In this paper, we are concerned with some spectral properties of the so-called pseudo p-Laplacian operator (see [4]), defined by

$$
\widetilde{\Delta}_{p} u:=\sum_{i=1}^{N} \frac{\partial}{\partial x_{i}}\left(\left|\frac{\partial u}{\partial x_{i}}\right|^{p-2} \frac{\partial u}{\partial x_{i}}\right),
$$

Where $1<p<\infty$. For $p=2$ this operator coincides with the usual Laplacian, but for $p \neq 2$ it considerably differs from the more familiar $p$-Laplace operator, given by

$$
\Delta_{p} u:=\sum_{i=1}^{N} \frac{\partial}{\partial x_{i}}\left(|\nabla u|^{p-2} \frac{\partial u}{\partial x_{i}}\right) .
$$


More precisely, we are concerned with investigating the Stekloff spectrum of $\widetilde{\Delta}_{p}$ on a generic open bounded Lipschitz set $\Omega \subset \mathbb{R}^{N}$, i.e. the set of those $\sigma \in \mathbb{R}$ such that the problem

$$
\begin{cases}\widetilde{\Delta}_{p} u=0, & \text { in } \Omega, \\ \sum_{i=1}^{N}\left|u_{x_{i}}\right|^{p-2} u_{x_{i}} \nu_{\Omega}^{i}=\sigma|u|^{p-2} u, & \text { on } \partial \Omega,\end{cases}
$$

admits nontrivial $W^{1, p}(\Omega)$ weak solutions. Here $\nu_{\Omega}=\left(\nu_{\Omega}^{1}, \ldots, \nu_{\Omega}^{N}\right)$ is the outer normal versor and we used the shortcut

$$
u_{x_{i}}:=\frac{\partial u}{\partial x_{i}} .
$$

It is easily seen that these numbers $\sigma$ can be equivalently characterized as the critical points of the restriction of the anisotropic p-Dirichlet integral

$$
\Phi_{p}(u)=\sum_{i=1}^{N} \int_{\Omega}\left|u_{x_{i}}\right|^{p} d x, \quad u \in W^{1, p}(\Omega),
$$

to the manifold $\left\{u \in W^{1, p}(\Omega):\|u\|_{L^{p}(\partial \Omega)}=1\right\}$.

\subsection{One step back: the linear case}

In order to smoothly present the topics and the aims of this paper, it could be useful to recall some basic facts from the linear case, i.e. we consider $p=2$ in (1.1). In this case, for every open bounded Lipschitz set $\Omega \subset \mathbb{R}^{N}$, its Stekloff eigenvalues of the Laplacian are the numbers $\sigma$ such that the problem

$$
\begin{cases}\Delta u=0, & \text { in } \Omega, \\ \left\langle\nabla u, \nu_{\Omega}\right\rangle=\sigma u, & \text { on } \partial \Omega,\end{cases}
$$

admits nontrivial $W^{1,2}(\Omega)$ solutions. By exploiting the compactness of the embedding $W^{1,2}(\Omega) \hookrightarrow L^{2}(\partial \Omega)$, it is classical to see that these eigenvalues form a discrete non-decreasing sequence diverging at $\infty$, i.e. $\sigma_{1}(\Omega) \leq \sigma_{2}(\Omega) \leq$ $\sigma_{3}(\Omega) \leq \cdots$, with $\sigma_{1}(\Omega)=0$ corresponding to constant solutions of (1.3). Again, these eigenvalues can be characterized as the critical values of the Dirichlet integral on the $L^{2}(\partial \Omega)$ unitary sphere. The corresponding critical points are the Stekloff eigenfunctions of $\Delta$ on $\Omega$, normalized by the condition on the $L^{2}(\partial \Omega)$ norm. Also, they turn out to form an orthonormal basis of $L^{2}(\partial \Omega)$. In particular, the first nontrivial eigenvalue $\sigma_{2}(\Omega)$ coincides with the value of the best constant in the following Poincaré-Wirtinger trace inequality

$$
c_{\Omega} \int_{\partial \Omega}\left|u-\bar{u}_{\partial \Omega}\right|^{2} d \mathcal{H}^{N-1} \leq \int_{\Omega}|\nabla u|^{2} d x, \quad u \in W^{1,2}(\Omega),
$$

where $\mathcal{H}^{N-1}$ stands for the $(N-1)$-dimensional Hausdorff measure and $\bar{u}_{\partial \Omega}$ is the average of (the trace of) $u$ on $\partial \Omega$.

In analogy with the well-known Dirichlet and Neumann cases (see [23, Chapters 3 and 7]), one may be interested in the spectral optimization problem of maximizing ${ }^{1} \sigma_{2}$ under volume constraint. A well-known result asserts that the (unique) solutions to this problem are given by balls. This is the so-called

\footnotetext{
1 On the contrary, it is not difficult to see that the problem of minimizing $\sigma_{2}$ is trivial.
} 
Brock-Weinstock inequality (see [9,33]). For ease of completeness, it is worth mentioning that Weinstock's result (valid only in dimension $N=2$ ) is even stronger, since it asserts that disks are still maximizers among simply connected set of given perimeter. By observing that $\sigma_{2}$ scales like a length to the power -1 and that $\sigma_{2}\left(B_{R}\right)=R^{-1}$ for a ball of radius $R$, the Brock-Weinstock inequality can be written in scaling invariant form as follows

$$
\sigma_{2}(\Omega) \leq\left(\frac{\omega_{N}}{|\Omega|}\right)^{\frac{1}{N}}
$$

where $\omega_{N}$ is the measure of the $N$-dimensional ball of radius 1 . Moreover, equality in (1.4) can hold if and only if $\Omega$ itself is a ball. Recently, a sharp quantitative stability estimate for this inequality has been given in [7, Corollary 5.2]. We recall that such an "isoperimetric" property of the ball is in turn a consequence of the fact that the ball (centered at the origin) is the only solution of

$$
\min \left\{\int_{\partial \Omega}|x|^{2} d \mathcal{H}^{N-1}: \Omega \subset \mathbb{R}^{N},|\Omega|=c\right\} .
$$

This result has been proved in [6, Theorem 4.2] (see also [7] for a different proof). In other words, we have the following weighted isoperimetric inequality

$$
\int_{\partial \Omega}|x|^{2} d \mathcal{H}^{N-1} \geq N \omega_{N}^{-1 / N}|\Omega|^{\frac{N+1}{N}},
$$

with equality if and only if $\Omega$ is a ball centered at the origin.

\subsection{Results and style of the paper}

Of course, problem (1.1) for $p \neq 2$ is a completely different story. We are now facing a nonlinear eigenvalue problem and no general results guarantee, for example, infiniteness and discreteness of the spectrum. Here we show that these eigenvalues form at least a countably infinite sequence of positive numbers $\sigma_{1, p}(\Omega) \leq \sigma_{2, p}(\Omega) \leq \sigma_{3, p}(\Omega) \leq \ldots$, diverging at infinity (Theorem 4.2). For this, we use some standard minimax methods from Nonlinear Analysis, but we will avoid to refer to the so-called Ljusternik-Schnirelmann theory and rather we will employ an alternative (and elegant) procedure introduced by Drabék and Robinson [15] (see the next section). Also, we will show that the first eigenvalue (again, we have $\sigma_{1, p}(\Omega)=0$ and it corresponds to constant eigenfunctions) is isolated in the spectrum, the latter being a closed set (Proposition 3.6). The first nontrivial eigenvalue $\sigma_{2, p}(\Omega)$ can still be characterized as the best constant in some Poincaré-Wirtinger trace inequality (Theorem 5.6 and Remark 5.7). Namely, $\sigma_{2, p}(\Omega)$ coincides with the best constant in

$$
c_{\Omega}\left[\min _{t \in \mathbb{R}} \int_{\partial \Omega}|u+t|^{p} d \mathcal{H}^{N-1}\right] \leq \sum_{i=1}^{N} \int_{\Omega}\left|u_{x_{i}}\right|^{p} d x, \quad u \in W^{1, p}(\Omega) .
$$

We will also address the issue of generalizing the Brock-Weinstock inequality, for the first nontrivial Stekloff eigenvalue of the pseudo $p$-Laplacian. Indeed, 
by adapting Brock's method of proof, we are able to prove (Theorems 7.2 and 7.3$)$

$$
\sigma_{2, p}(\Omega) \leq\left(\frac{\left|B_{p}\right|}{|\Omega|}\right)^{\frac{p-1}{N}}
$$

where $B_{p}$ is the $N$-dimensional $\ell^{p}$ unit ball, i.e. $B_{p}=\left\{x \in \mathbb{R}^{N}:\left|x_{1}\right|^{p}+\cdots+\right.$ $\left.\left|x_{N}\right|^{p}<1\right\}$. The previous inequality can be seen as a nonlinear counterpart of (1.4). Unfortunately, we are not able to detect the cases of equality in (1.6). More precisely, while it is rather easy to see that equality in (1.6) implies $\Omega$ to coincide with (a translated and scaled copy of) $B_{p}$, we can not guarantee that equality can really hold. This is due to the fact that we are not able to determine $\sigma_{2, p}\left(B_{p}\right)$ and this in turn is intimately linked to the lack of information on the shape of the nodal line of eigenfunctions corresponding to $\sigma_{2, p}\left(B_{p}\right)$ (see Remark 7.4). It is useful to recall at this point that even for the second eigenvalue of the $p$-Laplacian with Dirichlet boundary conditions, it is still an open problem to decide whether the nodal line in the Euclidean ball is given by a diameter (like in the linear case $p=2$ ) or not.

The proof of (1.6) is based on the anisotropic version of (1.5), which is here derived and appears to be new. Namely, we prove the following weighted Wulff inequality

$$
\int_{\partial \Omega}\|x\|^{\beta}\left\|\nu_{\Omega}\right\|_{*} d \mathcal{H}^{N-1} \geq N|K|^{\frac{1-\beta}{N}}|\Omega|^{\frac{N+\beta-1}{N}},
$$

where $\|\cdot\|$ and $\|\cdot\|_{*}$ are norms dual to each other, while $K$ is the unit ball of $\|\cdot\|$ centered at the origin and $\beta \geq 1$. Here again, equality can hold if and only if $\Omega=K$, up to a scaling factor. Inequality (1.7) is the other main contribution of this paper, its proof being an adaptation of the technique used in [7]. Since this result is not directly related with the study of the Stekloff spectrum, we added it in the Appendix at the end of the paper.

About the style of the paper, we point out that we tried to keep technicalities at a low level, in order to make the paper suitable for a wide audience, which can be possibly interested also in Isoperimetric Inequalities and Shape Optimization. Having this in mind, all the proofs, in despite of presenting many similarities with the vast literature on Nonlinear Spectral Theory, are as self-contained as possible. Further, sometimes our arguments are simpler and more direct. This is the case for example of the mountain pass characterization of the first nontrivial eigenvalue (Proposition 5.4), which is based on some peculiar convexity properties of the functional $\Phi_{p}$ in (1.2) (the so-called hidden convexity, see [5,8] and also [31]). Our method of proof still works for the standard $p$-Laplacian with Dirichlet or Neumann boundary conditions, thus it can be seen as alternative to that of [11, Theorem 3.1].

\subsection{A note on the regularity of eigenfunctions}

In passing, it is useful to say something about the regularity of Stekloff eigenfunctions of the pseudo $p$-Laplacian. We recall that in the case of the standard 
$p$-Laplacian operator, it is well-known that solutions to $\Delta_{p} u=0$ are $C^{1, \alpha}$ (see $[14,26])$. Then one could expect such a result to hold for $\widetilde{\Delta}_{p}$ as well.

However, we point out that the by now classical results of $[14,26]$ do not apply directly to the case of $\widetilde{\Delta}_{p}$, since the type of degeneracy is quite different. Low regularity (like $L^{\infty}$ or $C^{0, \alpha}$ ) is standard routine (see [22, Theorem 7.6]), due to the fact that pseudo $p$-harmonic functions are local minimizers of a functional having $p$-growth in the gradient variable. On the contrary, higher regularity is not clear. For example, to the best of our knowledge, even the (local) Lipschitz character of solutions seems not to be fully understood. We mention [5, Theorem 2.9 and Remark 2.11] where this is proved for the case $p \geq 2$, by using the theory of viscosity solutions (see also [32] for some pioneering results).

We also point out that in the case $1<p<2$ the existence of second weak derivatives for pseudo $p$-harmonic functions can still be proved, thus paralleling the case of the $p$-Laplacian (see [1]).

\subsection{Plan of the work}

We start with Sect. 2, where we recall some technical facts that will be useful throughout the whole paper. Then in Sect. 3 we introduce the Stekloff eigenvalue problem we are interested in and prove some first properties. We refine this study in Sect. 4, where the existence of an unbounded sequence of eigenvalues is exhibited. The subsequent Sect. 5 is devoted to the first nontrivial Stekloff eigenvalue. We prove some equivalent characterizations for it, which imply in particular that the first eigenvalue is always isolated in the spectrum. We further analyze the first nontrivial eigenspace in Sect. 6, proving nodal domains properties of eigenfunctions and giving yet another characterization of the first nontrivial eigenvalue, this time in terms of an eigenvalue problem with mixed boundary conditions. Finally, in Sect. 7 we provide some upper bounds for the first nontrivial eigenvalue, which can be seen as the nonlinear analogue of (1.4). As already said, a self-contained appendix devoted to weighted Wulff inequalities complements the paper.

\section{Technical machinery}

Given $1<p<\infty$, for every $x \in \mathbb{R}^{N}$ we will denote by $\|x\|_{\ell^{p}}$ its $\ell^{p}$ norm, i.e. the quantity

$$
\|x\|_{\ell^{p}}=\left(\sum_{i=1}^{N}\left|x_{i}\right|^{p}\right)^{\frac{1}{p}} .
$$

For an open set $\Omega \subset \mathbb{R}^{N}$, the symbol $W^{1, p}(\Omega)$ will stand for the usual Sobolev space, endowed with the norm

$$
\|u\|_{W^{1, p}(\Omega)}=\left(\int_{\Omega}|u|^{p} d x+\int_{\Omega}|\nabla u|^{p} d x\right)^{\frac{1}{p}} .
$$


As always, the symbol $W_{0}^{1, p}(\Omega)$ stands for the closure of $C_{0}^{\infty}(\Omega)$ with respect to the norm $\|\cdot\|_{W^{1, p}(\Omega)}$. It is useful to recall that when $\Omega \subset \mathbb{R}^{N}$ is bounded and has a Lipschitz boundary, the embedding $W^{1, p}(\Omega) \hookrightarrow L^{p}(\Omega)$ is compact. Moreover, each function in $W^{1, p}(\Omega)$ has a trace belonging to the fractional Sobolev spaces $W^{1-1 / p, p}(\partial \Omega)$. Then the embedding $W^{1, p}(\Omega) \hookrightarrow L^{p}(\partial \Omega)$ is compact as well.

Lemma 2.1. Let $1<p<\infty$ and $\Omega$ be an open bounded Lipschitz set in $\mathbb{R}^{N}$. Then

$$
\|\nabla u\|_{L^{p}(\Omega)}+\|u\|_{L^{p}(\partial \Omega)}, \quad u \in W^{1, p}(\Omega),
$$

defines a norm on the Sobolev space $W^{1, p}(\Omega)$, which is equivalent to (2.1).

Proof. It is straightforward to check that the above quantity defines a norm. Then the thesis is a consequence of the trace inequality

$$
\|u\|_{L^{p}(\partial \Omega)} \leq c_{\Omega}\|u\|_{W^{1, p}(\Omega)}
$$

and of the following Poincaré inequality

$$
\|u\|_{L^{p}(\Omega)} \leq \widetilde{c}_{\Omega}\left(\|\nabla u\|_{L^{p}(\Omega)}+\|u\|_{L^{p}(\partial \Omega)}\right),
$$

which in turn follows by a standard contradiction argument, exploiting the compact embedding $W^{1, p}(\Omega) \hookrightarrow L^{p}(\Omega)$.

The function $t \mapsto|t|^{p-2} t$ enjoys the following monotonicity properties, which will be useful in the sequel. For the proof, the reader is referred to [27, Section 10].

Lemma 2.2. Let $2 \leq p<\infty$. Then

$$
\left(|t|^{p-2} t-|s|^{p-2} s\right)(t-s) \geq 2^{2-p}|t-s|^{p}, \quad t, s \in \mathbb{R} .
$$

For $1<p<2$, we have

$$
\left(|t|^{p-2} t-|s|^{p-2} s\right)(t-s) \geq(p-1) \frac{|t-s|^{2}}{\left(1+|s|^{2}+|t|^{2}\right)^{\frac{2-p}{2}}}, \quad t, s \in \mathbb{R} .
$$

As an application of the previous Lemma, we obtain the following fact, which we will use repeatedly.

Proposition 2.3. Let $\left\{u^{k}\right\}_{k \in \mathbb{N}} \subset L^{p}(\Omega)$ be such that

$$
\lim _{k \rightarrow \infty} \int_{\Omega}\left(\left|u^{k}\right|^{p-2} u^{k}-|u|^{p-2} u\right)\left(u^{k}-u\right) d x=0
$$

for some function $u \in L^{p}(\Omega)$. Then $\left\{u^{k}\right\}_{k \in \mathbb{N}}$ converges in $L^{p}(\Omega)$ to $u$.

Proof. We have two distinguish between two cases. If $p \geq 2$, then by the first inequality of Lemma 2.2 , it follows directly that

$$
\lim _{k \rightarrow \infty} \int_{\Omega}\left|u^{k}-u\right|^{p} d x=0 .
$$

For $1<p<2$, we start observing that the hypothesis implies

$$
\int_{\Omega}\left|u^{k}\right|^{p} d x \leq C, \quad \text { for every } k \in \mathbb{N} \text {. }
$$


Indeed, by means of Young inequality we can infer

$$
\begin{aligned}
\int_{\Omega}\left(\left|u^{k}\right|^{p-2} u^{k}-|u|^{p-2} u\right)\left(u^{k}-u\right) d x \geq & \left(1-\frac{\varepsilon^{p}}{p}-\frac{\varepsilon^{q}}{q}\right) \int_{\Omega}\left|u^{k}\right|^{p} d x \\
& +\left(1-\frac{\varepsilon^{-p}}{p}-\frac{\varepsilon^{-q}}{q}\right) \int_{\Omega}|u|^{p} d x,
\end{aligned}
$$

where we set $q=p /(p-1)$. By taking $\varepsilon$ small enough and using (2.4), we then obtain (2.5). We now use inequality (2.3) of Lemma 2.2, raised to the power $p / 2$. Thus we get

$\int_{\Omega}\left|u^{k}-u\right|^{p} d x \leq C \int_{\Omega}\left(1+\left|u^{k}\right|^{2}+|u|^{2}\right)^{\frac{(2-p) p}{4}}\left[\left(\left|u^{k}\right|^{p-2} u^{k}-|u|^{p-2} u\right)\left(u^{k}-u\right)\right]^{\frac{p}{2}} d x$.

An application of Hölder inequality with exponents $2 /(2-p)$ and $2 / p$ yields

$$
\begin{aligned}
\int_{\Omega}\left|u^{k}-u\right|^{p} d x \leq & C\left[\int_{\Omega}\left(1+\left|u^{k}\right|^{2}+|u|^{2}\right)^{\frac{p}{2}} d x\right]^{\frac{2-p}{2}} \\
& \times\left[\int_{\Omega}\left(\left|u^{k}\right|^{p-2} u^{k}-|u|^{p-2} u\right)\left(u^{k}-u\right) d x\right]^{\frac{p}{2}} .
\end{aligned}
$$

The conclusion is now an easy consequence of (2.4) and (2.5).

To make the paper as self-contained as possible, we also recall the definition of Palais-Smale condition.

Definition 2.4. Let $\Phi: X \rightarrow \mathbb{R}$ be a $C^{1}$ functional defined on some Banach space $X$ and let $M \subset X$ be a $C^{1}$ manifold. Given $x \in M$, let us denote by $T_{x} M$ the tangent space at $M$ in the point $x$ and by $D \Phi(x)_{\mid T_{x} M}$ the differential in $x$ of the restriction of $\Phi$ to $M$.

Then $\Phi$ is said to satisfy the Palais-Smale condition on $M$ at level $\lambda$, if for every sequence $\left\{x_{n}\right\}_{n \in \mathbb{N}} \subset M$ such that

$$
\lim _{n \rightarrow \infty} \Phi\left(x_{n}\right)=\lambda \text { and } \lim _{n \rightarrow \infty}\left\|D \Phi\left(x_{n}\right)_{\mid T_{x_{n}} M}\right\|_{*}=0,
$$

we have that $x_{n}$ is strongly convergent, possibly up to the extraction of a subsequence. If this condition is verified for every $\lambda$, we will simply say that $\Phi$ satisfies the Palais-Smale condition on $M$.

In order to find critical points of functionals restricted to manifolds, in this paper we will use the following minimax procedure introduced by Drábek and Robinson [15] and further studied by Cuesta [10]. This is alternative to the so-called Ljusternik-Schnirelmann procedure, which relies on the concept of Krasnoselskii genus (see below). Our choice permits to produce a sufficiently large set of eigenvalues, avoiding at the same time unnecessary technicalities. The general result that we need is the following, due to Cuesta (see [10, Proposition 2.7] or [17] for the proof).

Theorem 2.5. Let $X$ be a uniformly convex Banach space and $M \subset X a C^{1}$ manifold. By indicating with $\mathbb{S}^{k-1}$ the unit sphere in $\mathbb{R}^{k}$, we set

$$
C_{o}\left(\mathbb{S}^{k-1} ; M\right)=\left\{f: \mathbb{S}^{k-1} \rightarrow M: f \text { is continuous and odd }\right\} .
$$


Suppose that $\Phi: X \rightarrow \mathbb{R}$ is an even $C^{1}$ functional and define

$$
\lambda_{k}=\inf _{f \in C_{o}\left(\mathbb{S}^{k-1} ; M\right)} \max _{u \in f\left(\mathbb{S}^{k-1}\right)} \Phi(u),
$$

If $\Phi$ verifies the Palais-Smale condition on $M$ at level $\lambda_{k}$, then $\lambda_{k}$ is a critical value of $\Phi$ on $M$, i.e. there exists $u_{0} \in M$ such that

$$
\Phi\left(u_{0}\right)=\lambda_{k} \quad \text { and } \quad D \Phi\left(u_{0}\right)_{\mid T_{u_{0}} M}=0 .
$$

Remark 2.6. For reader's convenience, we recall that the Krasnoselskii genus of a compact, nonempty and symmetric subset $A \subset X$ of a Banach space is defined by

$$
\gamma(A)=\inf \left\{k \in \mathbb{N}: \exists \text { a continuous odd mapp } f: A \rightarrow \mathbb{S}^{k-1}\right\},
$$

with the convention that $\gamma(A)=+\infty$, if no such an integer $k$ exists. Using the Krasnoselskii genus, an infinite sequence of critical values of $\Phi$ is usually produced as follows (see $[21,30])$

$$
\widetilde{\lambda}_{k}=\inf _{\gamma(A) \geq k} \max _{u \in A} \Phi(u), \quad k \in \mathbb{N} .
$$

It is an interesting open problem to decide whether or not the two previous minimax procedures give the same sets of critical values.

\section{The Stekloff spectrum of the pseudo $p$-Laplacian}

Let $\Omega \subset \mathbb{R}^{N}$ be an open bounded Lipschitz set and $\varrho: \partial \Omega \rightarrow \mathbb{R}$ be a measurable function satisfying

$$
0<c_{1} \leq \varrho(x) \leq c_{2}<\infty, \quad \mathcal{H}^{N-1} \text { - a.e. on } \partial \Omega .
$$

For every $1<p<\infty$, we consider the pseudo $p$-Laplacian, i.e. the nonlinear operator

$$
\widetilde{\Delta}_{p} u=\sum_{j=1}^{N}\left(\left|u_{x_{j}}\right|^{p-2} u_{x_{j}}\right)_{x_{j}} .
$$

Definition 3.1. A real number $\sigma$ is said to be a Stekloff eigenvalue of the pseudo p-Laplacian in $\Omega$ if the boundary value problem

$$
\begin{cases}\widetilde{\Delta}_{p} u=0, & \text { in } \Omega, \\ \sum_{i=1}^{N}\left|u_{x_{i}}\right|^{p-2} u_{x_{i}} \nu_{\Omega}^{i}=\sigma|u|^{p-2} u \varrho, & \text { on } \partial \Omega,\end{cases}
$$

admits a nontrivial solution $u$. If this is the case, we say that $u$ is a Stekloff eigenfunction corresponding to $\sigma$. We also set

$$
\mathfrak{S}_{p}(\Omega)=\{\sigma \in \mathbb{R}: \sigma \text { is a Stekloff eigenvalue }\},
$$

to denote the Stekloff spectrum of the pseudo $p$-Laplacian on $\Omega$.

Remark 3.2. Since the behaviour of the spectrum under varying weights is not investigated here, the notation does not account for the choice of the function $\varrho: \partial \Omega \rightarrow \mathbb{R}$. 
The solutions $u$ of the problem (3.2) are always understood in the weak sense, i.e. $u \in W^{1, p}(\Omega)$ and

$$
\sum_{i=1}^{N} \int_{\Omega}\left|u_{x_{i}}\right|^{p-2} u_{x_{i}} \varphi_{x_{i}} d x=\sigma \int_{\partial \Omega}|u|^{p-2} u \varphi \varrho d \mathcal{H}^{N-1}, \quad \text { for every } \varphi \in W^{1, p}(\Omega) .
$$

Observe that the integral on the right-hand side is well-defined, since the trace of a function in $W^{1, p}(\Omega)$ belongs to $L^{p}(\partial \Omega)$.

We start with the following basic result.

Lemma 3.3. Let $1<p<\infty, \Omega \subset \mathbb{R}^{N}$ be an open bounded Lipschitz set and $\varrho: \partial \Omega \rightarrow \mathbb{R}$ be such that (3.1) holds. There exists a least eigenvalue, given by $\sigma=0$ and corresponding to constant eigenfunctions. Moreover, every eigenfunction whose trace does not change sign on $\partial \Omega$ is constant in $\Omega$.

Proof. By testing with $\varphi=u$, equation (3.3) implies

$$
\int_{\Omega}\|\nabla u\|_{\ell^{p}}^{p} d x=\sigma \int_{\partial \Omega}|u|^{p} \varrho d \mathcal{H}^{N-1}
$$

so that every eigenvalue must be positive. Moreover, it is easily seen that $\sigma=0$ is an eigenvalue and by the previous equality any corresponding eigenfunction must be constant (on every connected component of $\Omega$ ).

Let us now prove the second part of the statement. Let $u \neq 0$ have a constant sign on the boundary and assume, arguing by contradiction, that it corresponds to an eigenvalue $\sigma \neq 0$. Inserting a constant test function in (3.3) we then obtain

$$
\int_{\partial \Omega}|u|^{p-1} \varrho d \mathcal{H}^{N-1}=0
$$

where we also used that $u$ does not change sign on $\partial \Omega$. Thus, $u$ has a null trace on $\partial \Omega$ and it solves in a weak sense the problem

$$
\left\{\begin{array}{cl}
\widetilde{\Delta}_{p} u=0 & \text { in } \Omega \\
u=0 & \text { on } \partial \Omega .
\end{array}\right.
$$

Solutions to the latter problem are minimizers of the strictly convex energy

$$
v \mapsto \int_{\Omega}\|\nabla v\|_{\ell^{p}}^{p} d x
$$

on $W_{0}^{1, p}(\Omega)$. Since the unique minimizer is given by the zero constant function, there must hold $u \equiv 0$, a contradiction. Therefore, $\sigma=0$ and $u$ is a constant eigenfunction.

Definition 3.4. If $u$ is a Stekloff eigenfunction, we will call nodal domains the connected components of $\{x \in \Omega: u(x) \neq 0\}$. Observe that the latter is an open set, since each pseudo $p$-harmonic function is locally Hölder continuous, as a local minimizer of $\int_{\Omega}\|\nabla v\|_{\ell^{p}}^{p}$ (see [22, Theorem 7.6]). We also observe that each nodal domain is itself an open set. This follows from the fact that the connected components of an open sets are open as well. 
As a straightforward consequence of Lemma 3.3, we get that every eigenfunction corresponding to an eigenvalue $\sigma>0$ has to change sign on the boundary. Then the following nodal domain property is immediate.

Lemma 3.5. Let $u \in W^{1, p}(\Omega)$ be a Stekloff eigenfunction, with eigenvalue $\sigma>$ 0. Then $u$ has at least two nodal domains, both touching the boundary.

We prove that the whole collection of Stekloff eigenvalues forms a closed set.

Proposition 3.6. Let $1<p<\infty, \Omega \subset \mathbb{R}^{N}$ a bounded Lipschitz domain and $\varrho: \partial \Omega \rightarrow \mathbb{R}$ be a function such that (3.1) holds. Then $\mathfrak{S}_{p}(\Omega)$ is a non empty closed subset of $[0, \infty)$.

Proof. The fact that the collection of all the Stekloff eigenvalues is non empty and consists of nonnegative numbers is due to Lemma 3.3. In order to prove the second part of the statement, we take a sequence of eigenvalues $\left\{\sigma^{k}\right\}_{k \in \mathbb{N}} \subset \mathfrak{S}_{p}(\Omega)$ converging to some positive number $\sigma$ and we let $\left\{u^{k}\right\}_{k \in \mathbb{N}} \subset$ $W^{1, p}(\Omega)$ be a sequence of corresponding eigenfunctions, normalized by the condition

$$
\int_{\partial \Omega}\left|u^{k}\right|^{p} \varrho d \mathcal{H}^{N-1}=1, \quad k \in \mathbb{N} .
$$

This implies in particular that

$$
\sum_{i=1}^{N} \int_{\Omega}\left|u_{x_{i}}^{k}\right|^{p} d x=\sigma^{k}, \quad k \in \mathbb{N},
$$

so that the sequence $\left\{u^{k}\right\}_{k \in \mathbb{N}}$ is bounded in $W^{1, p}(\Omega)$, thanks to Lemma 2.1. Thus, by the compactness of the embedding $W^{1, p}(\Omega) \hookrightarrow L^{p}(\partial \Omega)$, the sequence weakly converges (up to a subsequence) to some limit function $u$ in $W^{1, p}(\Omega)$. Moreover, this convergence is strong in $L^{p}(\partial \Omega)$. We have to show that $u$ is an eigenfuction with eigenvalue $\sigma$. By testing the equation solved by $u^{k}$ with $\varphi=u^{k}-u$, we then obtain

$$
\begin{aligned}
& \sum_{i=1}^{N} \int_{\Omega}\left(\left|u_{x_{i}}^{k}\right|^{p-2} u_{x_{i}}^{k}-\left|u_{x_{i}}\right|^{p-2} u_{x_{i}}\right)\left(u_{x_{i}}^{k}-u_{x_{i}}\right) d x \\
& \quad=\sigma_{k} \int_{\partial \Omega}\left|u^{k}\right|^{p-2} u^{k}\left(u^{k}-u\right) \varrho d \mathcal{H}^{N-1}-\sum_{i=1}^{N} \int_{\Omega}\left|u_{x_{i}}\right|^{p-2} u_{x_{i}}\left(u_{x_{i}}^{k}-u_{x_{i}}\right) d x .
\end{aligned}
$$

Then, by using that $\left\{u^{k}\right\}_{k \in \mathbb{N}}$ converges strongly in $L^{p}(\partial \Omega)$ and weakly in $W^{1, p}(\Omega)$, sending $k$ to infinity yields

$$
\lim _{k \rightarrow \infty} \sum_{i=1}^{N} \int_{\Omega}\left(\left|u_{x_{i}}^{k}\right|^{p-2} u_{x_{i}}^{k}-\left|u_{x_{i}}\right|^{p-2} u_{x_{i}}\right)\left(u_{x_{i}}^{k}-u_{x_{i}}\right) d x=0 .
$$

Thanks to Proposition 2.3, the previous gives the strong convergence of $\nabla u^{k}$ to $\nabla u$ in $L^{p}(\Omega)$. Since $\left\{u^{k}\right\}_{k \in \mathbb{N}}$ converges to $u$ strongly in $L^{p}(\partial \Omega)$, we also 
have

$$
\lim _{k \rightarrow \infty} \int_{\partial \Omega}\left|u^{k}-u\right|^{p} \varrho d \mathcal{H}^{N-1}=0 .
$$

Thanks to these informations, we can now pass to the limit in the equation (3.3) satisfied by $u^{k}$, so to obtain that $u$ is an eigenfunction as well, with eigenvalue $\sigma$. This shows that $\sigma \in \mathfrak{S}_{p}(\Omega)$, which is then closed.

\section{Existence of an unbounded sequence}

In this section, we will show that $\mathfrak{S}_{p}(\Omega)$ contains an infinite sequence of eigenvalues, diverging at $\infty$. At this aim, we observe that the elements of $\mathfrak{S}_{p}(\Omega)$ are the critical values of the functional

$$
\Phi(u)=\int_{\Omega}\|\nabla u\|_{\ell^{p}}^{p} d x, \quad u \in W^{1, p}(\Omega),
$$

on the manifold $M$, defined by

$$
M=\left\{u \in W^{1, p}(\Omega): G(u)=1\right\}, \quad \text { where } \quad G(u)=\int_{\partial \Omega}|u|^{p} \varrho d \mathcal{H}^{N-1} .
$$

Then, we will use the minimax procedure of Theorem 2.5. For this, we have to check that $\Phi$ verifies the Palais-Smale condition on $M$. This is the content of the next result.

Lemma 4.1. Let $\Omega \subset \mathbb{R}^{N}$ be an open bounded Lipschitz set and $\varrho: \partial \Omega \rightarrow \mathbb{R}$ be a function such that (3.1) holds. Then $\Phi$ satisfies the Palais-Smale condition on the manifold $M$.

Proof. First of all, we observe that $M$ is a $C^{1}$ manifold. It is sufficient to verify that 1 is a regular value for $G$, i.e. $D G(u) \neq 0$, for every $u \in M$. This is easily verified, since for every $u \in M$ we have

$$
D G(u)[u]=p \int_{\partial \Omega}|u|^{p} \varrho d \mathcal{H}^{N-1}=p \neq 0 .
$$

We now check that $\Phi$ satisfies the Palais-Smale condition on $M$. At this aim, we need to show that for every $C>0$ and every $\left\{u^{n}\right\}_{n \in \mathbb{N}} \subset M$, the following implication holds true

$$
\left.\begin{array}{l}
\Phi\left(u^{n}\right) \leq C \\
\lim _{n \rightarrow \infty}\left\|D \Phi\left(u^{n}\right)_{\mid T_{u^{n}} M}\right\|_{*}=0
\end{array}\right\} \Longrightarrow\left\{u^{n}\right\}_{n \in \mathbb{N}} \text { converges in } W^{1, p}(\Omega),
$$

Possibly up to the extraction of a subsequence. We first observe that for every $u \in M$, the tangent space to $M$ at the point $u$ is given by

$$
T_{u} M=\left\{\varphi \in W^{1, p}(\Omega): \int_{\partial \Omega}|u|^{p-2} u \varphi \varrho d \mathcal{H}^{N-1}=0\right\} .
$$


Hence, the hypothesis in (4.3) reads

$$
0=\lim _{n \rightarrow \infty}\left\|D \Phi\left(u^{n}\right)_{\mid T_{u^{n}} M}\right\|_{*}=\lim _{n \rightarrow \infty}\left[\sup _{\substack{\varphi \in T_{u^{n} M} \\ \varphi \neq 0}} \frac{\sum_{i=1}^{N} \int_{\Omega}\left|u_{x_{i}}^{n}\right|^{p-2} u_{x_{i}}^{n} \varphi_{x_{i}} d x}{\|\varphi\|_{W^{1, p}(\Omega)}}\right] .
$$

By this assumption, we can infer the existence of an infinitesimal sequence $\left\{\varepsilon_{n}\right\}_{n \in \mathbb{N}}$ of strictly positive numbers, such that

$$
\left.\left|\sum_{i=1}^{N} \int_{\Omega}\right| u_{x_{i}}^{n}\right|^{p-2} u_{x_{i}}^{n} \varphi_{x_{i}} d x \mid \leq \varepsilon_{n}\|\varphi\|_{W^{1, p}(\Omega)}, \quad \text { for every } \varphi \in T_{u^{n}} M .
$$

Observe that the sequence $\left\{u^{n}\right\}_{n \in \mathbb{N}}$ is bounded in $W^{1, p}(\Omega)$. Indeed, by using (3.1) and (4.3) we have

$$
\left\|u^{n}\right\|_{L^{p}(\partial \Omega)}^{p} \leq c_{1}^{-1} G\left(u^{n}\right)=c_{1}^{-1} \quad \text { and } \quad\left\|\nabla u^{n}\right\|_{L^{p}(\Omega)}^{p} \leq c \Phi\left(u^{n}\right) \leq \widetilde{C},
$$

and the boundedness follows by Lemma 2.1 .

Thus, by possibly passing to a subsequence, we can assume that $\left\{u^{n}\right\}_{n \in \mathbb{N}}$ converges weakly to a function $u$ in $W^{1, p}(\Omega)$ and that the convergence is strong both in $L^{p}(\Omega)$ and $L^{p}(\partial \Omega)$. In particular, such a limit function $u$ still belongs to $M$. Using the strong convergence in $L^{p}(\partial \Omega)$ again, we have

$$
\lim _{n \rightarrow \infty} \delta_{n}=0, \quad \text { where we set } \quad \delta_{n}=\int_{\partial \Omega}\left|u^{n}\right|^{p-2} u^{n}\left(u^{n}-u\right) \varrho d \mathcal{H}^{N-1} .
$$

Using the fact that $u^{n} \in M$, it is easy to check that the function $v^{n}:=$ $\left(1-\delta_{n}\right) u^{n}-u$ belongs to the tangent space $T_{u^{n}} M$. Thus, it is possible to choose $\varphi=v^{n}$ in (4.4), so as to obtain

$$
\begin{aligned}
\left.\left|\sum_{i=1}^{N} \int_{\Omega}\right| u_{x_{i}}^{n}\right|^{p-2} u_{x_{i}}^{n}\left(\left(1-\delta_{n}\right) u_{x_{i}}^{n}-u_{x_{i}}\right) d x \mid & \leq \varepsilon_{n}\left\|\left(1-\delta_{n}\right) u^{n}-u\right\|_{W^{1, p}(\Omega)} \\
& \leq \varepsilon_{n}\left(2\left\|u^{n}\right\|_{W^{1, p}(\Omega)}+\|u\|_{W^{1, p}(\Omega)}\right),
\end{aligned}
$$

where we used that $\left|1-\delta_{n}\right| \leq 2$ by (4.5), provided $n$ is large enough. By passing to the limit as $n \rightarrow \infty$, we then get

$$
\lim _{n \rightarrow \infty} \sum_{i=1}^{N} \int_{\Omega}\left|u_{x_{i}}^{n}\right|^{p-2} u_{x_{i}}^{n}\left(\left(1-\delta_{n}\right) u_{x_{i}}^{n}-u_{x_{i}}\right)=0,
$$

and so

$$
\lim _{n \rightarrow \infty} \sum_{i=1}^{N} \int_{\Omega}\left|u_{x_{i}}^{n}\right|^{p-2} u_{x_{i}}^{n}\left(u_{x_{i}}^{n}-u_{x_{i}}\right) d x=0 .
$$

Since $u^{n}$ weakly converges to $u$ in $W^{1, p}(\Omega)$, we also have

$$
\lim _{n \rightarrow \infty} \sum_{i=1}^{N} \int_{\Omega}\left|u_{x_{i}}\right|^{p-2} u_{x_{i}}\left(u_{x_{i}}^{n}-u_{x_{i}}\right) d x=0 .
$$


By subtracting (4.7) to (4.6) and using again Proposition 2.3 on each component $u_{x_{i}}^{n}$, we then get

$$
\lim _{n \rightarrow \infty} \int_{\Omega}\left|\nabla\left(u^{n}-u\right)\right|^{p} d x=0 .
$$

By owing to Lemma 2.1, finally the strong convergence of $\left\{u^{n}\right\}_{n \in \mathbb{N}}$ in $W^{1, p}(\Omega)$ follows by (4.8). This shows the validity of the implication (4.3), thus concluding the proof.

We can then assure that $\mathfrak{S}_{p}(\Omega)$ contains an unbounded sequence. In the following, we keep the same notation as in Sect. 2 .

Theorem 4.2. Given $1<p<\infty$, let $\Omega \subset \mathbb{R}^{N}$ be an open bounded connected set, having Lipschitz boundary. Let also $\varrho: \partial \Omega \rightarrow \mathbb{R}$ be a function such that (3.1) holds. For every $k \in \mathbb{N}$, we define

$$
\sigma_{k, p}(\Omega)=\inf _{f \in C_{o}\left(\mathbb{S}^{k-1} ; M\right)} \max _{u \in f\left(\mathbb{S}^{k-1}\right)} \int_{\Omega}\|\nabla u\|_{\ell^{p}}^{p} d x .
$$

Then each $\sigma_{k, p}(\Omega)$ is a Stekloff eigenvalue of the pseudo p-Laplacian on $\Omega$. Moreover,

$$
0=\sigma_{1, p}(\Omega)<\sigma_{2, p}(\Omega) \leq \cdots \leq \sigma_{k, p}(\Omega) \leq \cdots
$$

and $\sigma_{k, p}(\Omega) \rightarrow \infty$ as $k \rightarrow \infty$.

Proof. For the sake of readability, we divide the proof in various steps.

Eigenvalues We already observed that the Stekloff eigenvalues of the pseudo $p$-Laplacian are precisely the critical values of the functional (4.1) on the $C^{1}$ manifold $M$. It is then sufficient to observe that the assumptions of Theorem 2.5 are satisfied, thanks to Lemma 4.1. Hence the first part of the statement is proved.

The sequence is non-decreasing Let $k \in \mathbb{N}$ and $f: \mathbb{S}^{k} \rightarrow M$ be an odd continuous mapping. Then, we take the vector subspace $E=\left\{x=\left(x_{1}, \ldots, x_{k+1}\right) \in\right.$ $\left.\mathbb{R}^{k+1}: x_{k+1}=0\right\}$ and we consider the restriction $f_{E}$ of $f$ to the intersection $\mathbb{S}^{k} \cap E \simeq \mathbb{S}^{k-1}$. Whence

$$
\begin{aligned}
\max _{u \in f\left(\mathbb{S}^{k}\right)} \int_{\Omega}\|\nabla u\|_{\ell^{p}}^{p} d x & \geq \max _{u \in f\left(\mathbb{S}^{k} \cap E\right)} \int_{\Omega}\|\nabla u\|_{\ell^{p}}^{p} d x \\
& =\max _{u \in f_{E}\left(\mathbb{S}^{k-1}\right)} \int_{\Omega}\|\nabla u\|_{\ell^{p}}^{p} d x \\
& \geq \inf _{g \in C_{o}\left(\mathbb{S}^{k-1} ; M\right)} \max _{u \in g\left(\mathbb{S}^{k-1}\right)} \int_{\Omega}\|\nabla u\|_{\ell^{p}}^{p} d x=\sigma_{k, p}(\Omega) .
\end{aligned}
$$

Since the mapping $f$ was arbitrarily chosen, passing to the infimum we get

$$
\sigma_{k+1, p}(\Omega) \geq \sigma_{k, p}(\Omega),
$$

as desired.

The first element is zero We show that the first element of the sequence (4.9) is in fact zero. To this end, we note that any continuous odd mapping from $\mathbb{S}^{0}=\{1,-1\}$ to $M$ can be identified with the choice of an antipodal pair $\{u,-u\}$ on the symmetric manifold $M$. This and the fact that the functional 
is even imply that if $k=1$ formula (4.9) gives the minimum of (4.1) on $M$. The latter is of course zero, corresponding to constant functions.

Existence of a gap. We prove that there is a gap between zero and the second term of the sequence, i.e. $\sigma_{2, p}(\Omega)>0$. At this aim, let us argue by contradiction and suppose that

$$
\sigma_{2, p}(\Omega)=\inf _{f \in C_{o}\left(\mathbb{S}^{1} ; M\right)} \max _{u \in f\left(\mathbb{S}^{1}\right)} \int_{\Omega}\|\nabla u\|_{\ell^{p}}^{p} d x=0,
$$

so that, for all $n \in \mathbb{N}$ there exists an odd continuous mapping $f_{n}: \mathbb{S}^{1} \rightarrow M$ such that

$$
\max _{u \in f_{n}\left(\mathbb{S}^{1}\right)} \int_{\Omega}\|\nabla u\|_{\ell^{p}}^{p} d x \leq \frac{1}{n} .
$$

Since $\Omega$ is connected, we observe that

$$
c=\left(\int_{\partial \Omega} \varrho d \mathcal{H}^{N-1}\right)^{-\frac{1}{p}} \cdot 1_{\Omega}
$$

defines the unique (modulo the choice of the sign) constant function belonging to $M$. Let $0<\varepsilon<1 / 2$ and consider the two neighborhoods

$B_{\varepsilon}^{+}=\left\{u \in M:\|u-c\|_{L^{p}(\partial \Omega, \varrho)}<\varepsilon\right\} \quad B_{\varepsilon}^{-}=\left\{u \in M:\|u-(-c)\|_{L^{p}(\partial \Omega, \varrho)}<\varepsilon\right\}$, which are disjoint, by construction. Here we set for brevity

$$
\|\varphi\|_{L^{p}(\partial \Omega ; \varrho)}=\left(\int_{\partial \Omega}|\varphi|^{p} \varrho d \mathcal{H}^{N-1}\right)^{\frac{1}{p}} .
$$

Since the mapping $f_{n}$ is odd and continuous, for every $n \in \mathbb{N}$ the image $f_{n}\left(\mathbb{S}^{1}\right)$ is symmetric and connected, then it can not be contained in $B_{\varepsilon}^{+} \cup B_{\varepsilon}^{-}$, the latter being symmetric and disconnected. So we can pick an element

$$
u_{n} \in f_{n}\left(\mathbb{S}^{1}\right) \backslash\left(B_{\varepsilon}^{+} \cup B_{\varepsilon}^{-}\right) .
$$

This yields a sequence $\left\{u_{n}\right\}_{n \in \mathbb{N}} \subset M$, which is bounded in $W^{1, p}(\Omega)$ by (4.11). Hence, there exists a function $v \in M$ such that $\left\{u_{n}\right\}_{n \in \mathbb{N}}$ converges to $v$ weakly in $W^{1, p}(\Omega)$ and strongly in $L^{p}(\partial \Omega)$, possibly by passing to a subsequence. By the weak convergence it follows that

$$
\int_{\Omega}\|\nabla v\|_{\ell^{p}}^{p} d x \leq \liminf _{n \rightarrow \infty} \int_{\Omega}\left\|\nabla u_{n}\right\|_{\ell^{p}}^{p} d x \leq \lim _{n \rightarrow \infty} \frac{1}{n}=0 .
$$

As $\Omega$ is connected, this in turn shows that $v \in M$ is constant in $\Omega$, so that either $v=c$ or $v=-c$. Let us assume that $v=c$, for example: by using the strong convergence in $L^{p}(\partial \Omega)$, we get

$$
0=\|v-c\|_{L^{p}(\partial \Omega, \varrho)}=\lim _{n \rightarrow \infty}\left\|u_{n}-c\right\|_{L^{p}(\partial \Omega, \varrho)} \geq \varepsilon,
$$

where in the last inequality we used (4.12). This gives a contradiction and thus $\sigma_{2, p}(\Omega)>0$.

Unboundedness It remains to prove that this sequence of eigenvalues is unbounded. To this aim, we suitably modified the argument of [21, Proposition 5.4] (for a different proof, avoiding the use of Schauder bases, one could adapt the argument of [18, Theorem 5.2]). 
We recall that the Sobolev space $W^{1, p}(\Omega)$ admits a Schauder basis (see $[19,28])$. Hence, there exists an ordered countable set of elements $\left\{e_{n}\right\}_{n \in \mathbb{N}} \subset$ $W^{1, p}(\Omega)$ with the property that for all $u \in W^{1, p}(\Omega)$, we have

$$
u=\sum_{n=1}^{\infty} \alpha_{n} e_{n}
$$

for a (uniquely determined) sequence of coefficients $\left\{\alpha_{n}\right\}_{n \in \mathbb{N}}$. Here the convergence of the previous series has to be understood in the sense of the norm topology. Clearly, if we now denote by

$$
E_{n}=\operatorname{Vect}\left(\left\{e_{1}, \ldots, e_{n}\right\}\right),
$$

the linear envelope of the first $n$ elements of the basis, then the union $\bigcup_{n \in \mathbb{N}} E_{n}$ is dense in $W^{1, p}(\Omega)$. Let us also set

$$
F_{k}=\overline{\operatorname{Vect}\left(\left\{e_{n}\right\}_{n>k}\right)},
$$

which is the topological supplement of the finite-dimensional vector space $E_{k}$, and define the new sequence

$$
\tau_{k, p}(\Omega)=\inf _{f \in C_{o}\left(\mathbb{S}^{k-1} ; M\right)} \max _{u \in f\left(\mathbb{S}^{k-1}\right) \cap F_{k-1}} \int_{\Omega}\|\nabla u\|_{\ell^{p}}^{p} d x, \quad k \in \mathbb{N} .
$$

At first, we verify that such a sequence is actually well defined. Indeed, let $f$ be an odd and continuous map from the unit sphere $\mathbb{S}^{k-1}$ to $M$ and assume that the intersection $f\left(\mathbb{S}^{k-1}\right) \cap F_{k-1}$ is empty: this implies that for every $\omega \in \mathbb{S}^{k-1}$, the element $f(\omega)$ always has at least a nontrivial component on $E_{k-1}$. By composing $f$ with the continuous odd operator

$$
P_{k-1}: W^{1, p}(\Omega) \rightarrow E_{k-1},
$$

given by the natural projection on the linear space $E_{k-1}$, the map $P_{k-1} \circ f$ is odd, continuous and $P_{k-1} \circ f(\omega) \neq 0$, for every $\omega \in \mathbb{S}^{k-1}$. That is, we constructed an odd continuous map from $\mathbb{S}^{k-1}$ to $E_{k-1} \backslash\{0\} \simeq \mathbb{R}^{k-1} \backslash\{0\}$ and this contradicts the Borsuk-Ulam Theorem ${ }^{2}$. Hence the image of any $f \in$ $C_{o}\left(\mathbb{S}^{k-1} ; M\right)$ has to intersect $F_{k-1}$, for every $k \in \mathbb{N}$.

Since obviously $\tau_{k, p}(\Omega) \leq \sigma_{k, p}(\Omega)$, it sufficies now to show that

$$
\lim _{k \rightarrow \infty} \tau_{k, p}(\Omega)=+\infty \text {. }
$$

At this aim, assume by contradiction that $\tau_{k, p}(\Omega)<\tau$, for all $k \in \mathbb{N}$. Then, for every $k \in \mathbb{N}$, we can take a mapping $f \in C_{o}\left(\mathbb{S}^{k} ; M\right)$ and $u_{k} \in f\left(\mathbb{S}^{k-1}\right) \cap F_{k-1}$ such that

$$
\int_{\Omega}\left\|\nabla u_{k}\right\|_{\ell^{p}}^{p} d x<\tau
$$

Since $u_{k} \in M$ for all $k \in \mathbb{N}$, equation (4.13) implies that the sequence $\left\{u_{k}\right\}_{k \in \mathbb{N}}$ is bounded in $W^{1, p}(\Omega)$ and weakly converges (up to a subsequence) to some limit function $u \in M$.

\footnotetext{
${ }^{2}$ We recall that the Borsuk-Ulam states the following: "for every continuous map $f: \mathbb{S}^{k} \rightarrow$ $\mathbb{R}^{k}$, there exists $x_{0} \in \mathbb{S}^{k}$ such that $f\left(x_{0}\right)=f\left(-x_{0}\right)$ ". Since our function $P_{k-1} \circ f$ is odd, this would give that $0 \in \operatorname{Im}\left(P_{k-1} \circ f\right)$, that is a contradiction.
} 
On the other hand, by definition of Schauder basis, the functionals defined on $W^{1, p}(\Omega)$ by

$$
\phi_{k}(u)=\alpha_{k}, \quad \text { if } \quad u=\sum_{n=1}^{+\infty} \alpha_{n} e_{n} \in W^{1, p}(\Omega), \quad k \in \mathbb{N},
$$

are linear and they turn out to be continuous, thanks to [3, page 83]. By the weak convergence of the sequence $\left\{u_{k}\right\}_{k \in \mathbb{N}}$ to $u$, it follows that $\lim _{k \rightarrow \infty} \phi_{n}\left(u_{k}\right)=\phi_{n}(u)$, for all $n \in \mathbb{N}$. Since $u_{k} \in F_{k-1}$, we have that

$$
\phi_{n}\left(u_{k}\right)=0, \quad \text { for every } n \leq k-1,
$$

thus $\phi_{n}(u)=0$ for all $n \in \mathbb{N}$. This means that $u=0$, contradicting the fact that $u \in M$.

Remark 4.3. If $\Omega$ has $m$ connected components $\Omega_{1}, \ldots, \Omega_{m}$, equation (4.9) still defines an infinite sequence of eigenvalues diverging at $\infty$, but in this case we have

$$
\sigma_{1, p}(\Omega)=\cdots=\sigma_{m, p}(\Omega)=0,
$$

corresponding to the (normalized) piecewise constant eigenfunctions

$$
c_{i}=\left(\int_{\partial \Omega_{i}} \varrho d \mathcal{H}^{N-1}\right)^{-\frac{1}{p}} \cdot 1_{\Omega_{i}}(x) .
$$

\section{The first nontrivial eigenvalue}

We are going to show that $\sigma_{2, p}(\Omega)$ is actually the first nontrivial Stekloff eigenvalue of $\widetilde{\Delta}_{p}$. In other words, the first eigenvalue $\sigma=0$ is always isolated in the spectrum and any other eigenvalue has to be greater than $\sigma_{2, p}(\Omega)$. Then the quantity $\sigma_{2, p}(\Omega)$ can also be seen as the fundamental gap of the pseudo $p$-Laplacian, with Stekloff boundary conditions.

Theorem 5.1. Let $u \in W^{1, p}(\Omega)$ be a Stekloff eigenfunction, with eigenvalue $\sigma>0$. Then we have $\sigma \geq \sigma_{2, p}(\Omega)$.

Proof. The proof is inspired to [24, Theorem 3.4]. At first, we observe that the positive and negative parts

$$
u_{+}(x)=\max \{u(x), 0\} \quad \text { and } \quad u_{-}(x)=\max \{0,-u(x)\},
$$

are both not identically zero, thanks to Lemma 3.5. Also, they still belong to $W^{1, p}(\Omega)$, hence they have a trace on the boundary $\partial \Omega$. Moreover, we have

$$
\operatorname{trace}_{\mid \partial \Omega}\left(u_{+}\right)=\left(\operatorname{trace}_{\mid \partial \Omega} u\right)_{+} \text {and } \operatorname{trace}_{\mid \partial \Omega}\left(u_{-}\right)=\left(\operatorname{trace}_{\mid \partial \Omega} u\right)_{-},
$$

so that by using $u_{+}$and $u_{-}$as test functions in (3.3), we obtain

$$
\int_{\Omega}\left\|\nabla u_{+}\right\|_{\ell^{p}}^{p} d x=\sigma \int_{\partial \Omega}\left|u_{+}\right|^{p} \varrho d \mathcal{H}^{N-1},
$$

and

$$
\int_{\Omega}\left\|\nabla u_{-}\right\|_{\ell^{p}}^{p} d x=\sigma \int_{\partial \Omega}\left|u_{-}\right|^{p} \varrho d \mathcal{H}^{N-1}
$$


Let us now consider the odd and continuous mapping $\tilde{f}: \mathbb{S}^{1} \rightarrow M$ defined by

$$
\begin{aligned}
\tilde{f}_{\omega}(x) & =\frac{\omega_{1} u_{+}(x)-\omega_{2} u_{-}(x)}{\left(\left|\omega_{1}\right|^{p} \int_{\partial \Omega}\left|u_{+}\right|^{p} \varrho d \mathcal{H}^{N-1}+\left|\omega_{2}\right|^{p} \int_{\partial \Omega}\left|u_{-}\right|^{p} \varrho d \mathcal{H}^{N-1}\right)^{1 / p}}, \\
\omega & =\left(\omega_{1}, \omega_{2}\right) \in \mathbb{S}^{1} .
\end{aligned}
$$

If we choose $f=\tilde{f}$ in the definition of $\sigma_{2, p}(\Omega)$, we then get

$$
\sigma_{2, p}(\Omega) \leq \max _{\omega \in \mathbb{S}^{1}} \frac{\left|\omega_{1}\right|^{p} \int_{\Omega}\left\|\nabla u_{+}\right\|_{\ell^{p}}^{p} d x+\left|\omega_{2}\right|^{p} \int_{\Omega}|| \nabla u_{-} \|_{\ell^{p}}^{p} d x}{\left|\omega_{1}\right|^{p} \int_{\partial \Omega}\left|u_{+}\right|^{p} \varrho d \mathcal{H}^{N-1}+\left|\omega_{2}\right|^{p} \int_{\partial \Omega}\left|u_{-}\right|^{p} \varrho d \mathcal{H}^{N-1}}=\sigma,
$$

concluding thus the proof.

We devote the rest of this section to give some alternative characterizations of $\sigma_{2, p}(\Omega)$. The first one is a mountain pass characterization. The main tool we need is the following Lemma, which guarantees that we can always join functions on

$$
M=\left\{u \in W^{1, p}(\Omega): \int_{\partial \Omega}|u|^{p} \varrho d \mathcal{H}^{N-1}=1\right\},
$$

by a curve on which the energy $\int_{\Omega}\|\nabla u\|_{\ell^{p}}^{p}$ is controlled by the values at the endpoints. For this, some assumptions on the sign of functions involved are necessary.

Lemma 5.2. Let $u, v \in M$, with $v \geq 0$ on $\Omega$ and $u$ satisfying one of the following assumptions:

(i) $u \geq 0$ on $\Omega$;

(iii) the positive and negative parts of $u$ are both not identically zero and

$$
u_{+} \not \equiv 0 \text { on } \partial \Omega \quad \text { and } \quad \frac{\int_{\Omega}\left\|\nabla u_{+}\right\|_{\ell^{p}}^{p} d x}{\int_{\partial \Omega} u_{+}^{p} \varrho d \mathcal{H}^{N-1}} \leq \frac{\int_{\Omega}\left\|\nabla u_{-}\right\|_{\ell^{p}}^{p} d x}{\int_{\partial \Omega} u_{-}^{p} \varrho d \mathcal{H}^{N-1}}
$$

with the convention that (5.1) is satisfied if $u_{-} \equiv 0$ on $\partial \Omega$.

Then there exists a continuous curve $\gamma:[0,1] \rightarrow M$ connecting $u$ and $v$, such that

$$
\int_{\Omega}\left\|\nabla \gamma_{t}\right\|_{\ell^{p}}^{p} d x \leq \max \left\{\int_{\Omega}\|\nabla u\|_{\ell^{p}}^{p} d x, \int_{\Omega}\|\nabla v\|_{\ell^{p}}^{p} d x\right\}, \quad t \in[0,1] .
$$

Proof. If $u$ is positive on $\Omega$, in order to conclude it sufficies to observe that the anisotropic Dirichlet integral is convex along the curve defined by

$$
\gamma_{t}(x)=\left((1-t) u(x)^{p}+t v(x)^{p}\right)^{\frac{1}{p}}, \quad x \in \Omega, t \in[0,1] .
$$

Namely, inequality

$$
\int_{\Omega}\left\|\nabla \gamma_{t}\right\|_{\ell^{p}}^{p} d x \leq(1-t) \int_{\Omega}\|\nabla u\|_{\ell^{p}}^{p} d x+t \int_{\Omega}\|\nabla v\|_{\ell^{p}}^{p} d x,
$$


holds for all $t \in[0,1]$, by owing to [8, Lemma 2.1]. We point out that the image of such a curve $\gamma$ lives on $M$ and is indeed continuous in the norm topology of $W^{1, p}(\Omega)$ (see [17, Lemma 4.1.1]).

Let us now suppose that $u_{+}$and $u_{-}$are both non identically zero on $\Omega$ and that (5.1) holds. Set

$$
\sigma_{t}(x)=\frac{u_{+}(x)-\cos (\pi t) u_{-}(x)}{\left\|u_{+}-\cos (\pi t) u_{-}\right\|_{L^{p}(\partial \Omega, \varrho)}}, \quad t \in\left[0, \frac{1}{2}\right] .
$$

Then $\sigma_{t}$ is a continuous curve on $M$, connecting $u$ to its (renormalized) positive part. Since $u_{+}$and $u_{-}$have disjoint supports, we get

$$
\int_{\Omega}\left\|\nabla \sigma_{t}\right\|_{\ell^{p}}^{p} d x=\frac{\int_{\Omega}\left\|\nabla u_{+}\right\|_{\ell^{p}}^{p} d x+|\cos (\pi t)|^{p} \int_{\Omega}\left\|\nabla u_{-}\right\|_{\ell^{p}}^{p} d x}{\int_{\partial \Omega} u_{+}^{p} \varrho d \mathcal{H}^{N-1}+|\cos (\pi t)|^{p} \int_{\partial \Omega} u_{-}^{p} \varrho d \mathcal{H}^{N-1}}
$$

whence

$$
\int_{\Omega}\left\|\nabla \sigma_{t}\right\|_{\ell^{p}}^{p} d x \leq \int_{\Omega}\left\|\nabla \sigma_{0}\right\|_{\ell^{p}}^{p} d x=\int_{\Omega}\|\nabla u\|_{\ell^{p}}^{p} d x,
$$

for all $t \in\left[0, \frac{1}{2}\right]$, by $(5.1)$ and basic calculus ${ }^{3}$. In order to conclude, it is now sufficient to connect the (renormalized) positive part of $u$ to $v$. For this, we can simply use a curve like (5.2), suitably reparametrized, i.e.

$$
\widetilde{\sigma}_{t}(x)=\left((2-2 t) \frac{u_{+}(x)^{p}}{\left\|u_{+}\right\|_{L^{p}(\partial \Omega, \varrho)}^{p}}+(2 t-1) v(x)^{p}\right)^{\frac{1}{p}}, \quad t \in\left[\frac{1}{2}, 1\right],
$$

and exploit once again the convexity of the functional along this curve. Finally, by gluing together the two curves, i.e. by defining

$$
\gamma_{t}(x)=\sigma_{t}(x), \quad t \in\left[0, \frac{1}{2}\right] \quad \text { and } \quad \gamma_{t}(x)=\widetilde{\sigma}_{t}(x), \quad t \in\left[\frac{1}{2}, 1\right],
$$

we get the desired conclusion. Observe that the same construction above is still feasible if $u_{-} \equiv 0$ on $\partial \Omega$.

Remark 5.3. Of course, the positivity of the function $v$ in the previous Lemma can be dropped and replaced by condition (5.1). We kept it just for ease of exposition.

Given a pair of functions $u, v \in M$, we denote by $\Gamma_{\Omega}(u, v)$ the set of all continuous paths in $M$, parametrized on $[0,1]$ and connecting $u$ to $v$, i.e.

$$
\Gamma_{\Omega}(u, v)=\{\gamma:[0,1] \rightarrow M: \gamma \text { is continuous and } \gamma(0)=u, \gamma(1)=v\},
$$

where continuity is understood in the norm topology of $W^{1, p}$. Then we have the following alternative characterization for $\sigma_{2, p}(\Omega)$.

${ }^{3}$ We use the simple fact that

$$
h(s)=\frac{a^{2}+s b^{2}}{c^{2}+s d^{2}}, \quad s \in \mathbb{R},
$$

is increasing if $b^{2} / d^{2} \geq a^{2} / c^{2}$, so that in particular $h(s) \leq h(1)$ for every $s \in[0,1]$. 
Proposition 5.4. Let $\Omega \subset \mathbb{R}^{N}$ be an open bouned connected Lipschitz set. Let us define the constant function

$$
c=\left(\int_{\partial \Omega} \varrho d \mathcal{H}^{N-1}\right)^{-\frac{1}{p}} \in M .
$$

Then the first nontrivial Stekloff eigenvalue has the following Mountain Pass characterization

$$
\sigma_{2, p}(\Omega)=\inf _{\gamma \in \Gamma_{\Omega}(c,-c)} \max _{u \in \gamma} \int_{\Omega}\|\nabla u\|_{\ell^{p}}^{p} d x .
$$

Proof. For every $\gamma \in \Gamma_{\Omega}(c,-c)$, the closed path on $M$ obtained by gluing $\gamma$ and $-\gamma$ is in fact the image of some odd continuous mapping $f_{\gamma}$ from $\mathbb{S}^{1}$ to $M$. Hence, by definition of $\sigma_{2, p}(\Omega)$ we get

$$
\sigma_{2, p}(\Omega) \leq \max _{u \in f_{\gamma}\left(\mathbb{S}^{1}\right)} \int_{\Omega}\|\nabla u\|_{\ell^{p}}^{p} d x=\max _{u \in \gamma} \int_{\Omega}\|\nabla u\|_{\ell^{p}}^{p} d x .
$$

By taking the infimum among all admissible paths $\gamma$, we easily obtain

$$
\sigma_{2, p}(\Omega) \leq \inf _{\gamma \in \Gamma_{\Omega}(-c, c)} \max _{u \in \gamma} \int_{\Omega}\|\nabla u\|_{\ell^{p}}^{p} d x .
$$

To prove the reverse inequality, we proceed as follows: for every $n \in \mathbb{N}$, let us take $\left\{f_{n}\right\}_{n \in \mathbb{N}} \subset C_{o}\left(\mathbb{S}^{1} ; M\right)$ such that

$$
\max _{u \in f_{n}\left(\mathbb{S}^{1}\right)} \int_{\Omega}\|\nabla u\|_{\ell^{p}}^{p} d x \leq \sigma_{2, p}(\Omega)+\frac{1}{n} .
$$

Let us then pick up $u^{n} \in f_{n}\left(\mathbb{S}^{1}\right)$ such that one of the hypotheses of Lemma 5.2 is satisfied ${ }^{4}$. Then we can assure the existence of a continuous curve $\sigma^{n}$ on $M$, connecting the constant function $c$ to $u^{n}$ and such that

$$
\int_{\Omega}\left\|\nabla \sigma_{t}^{n}\right\|_{\ell^{p}}^{p} d x \leq \int_{\Omega}\left\|\nabla u^{n}\right\|_{\ell^{p}}^{p} d x \leq \sigma_{2, p}(\Omega)+\frac{1}{n} .
$$

Symmetrically, the path $-\sigma^{n}$ connects $-c$ to $-u^{n} \in f_{n}\left(\mathbb{S}^{k-1}\right)$ and the previous estimate still holds true on this path, since the functional is even. Then gluing together the three paths $\sigma^{n},-\sigma^{n}$ and $f_{n}$, we get a continuous curve $\Sigma^{n} \in \Gamma_{\Omega}(c,-c)$ such that

$$
\max _{u \in \Sigma^{n}} \int_{\Omega}\|\nabla u\|_{\ell^{p}}^{p} d x \leq \sigma_{2, p}(\Omega)+\frac{1}{n} .
$$

Passing to the infimum over $\Gamma_{\Omega}(c,-c)$, we then get

$$
\inf _{\gamma \in \Gamma_{\Omega}(c,-c)} \max _{u \in \gamma} \int_{\Omega}\|\nabla u\|_{\ell^{p}}^{p} d x \leq \sigma_{2, p}(\Omega)+\frac{1}{n} .
$$

By letting $n$ tends to $\infty$, we finally get the desired result.

\footnotetext{
${ }^{4}$ Observe that it is always possible to make such a choice, since $f_{n}\left(\mathbb{S}^{k-1}\right)$ is symmetric, i.e. if $u \in f_{n}\left(\mathbb{S}^{k-1}\right)$, then $-u \in f_{n}\left(\mathbb{S}^{k-1}\right)$ as well. Moreover, a function $u \in M$ can not have a zero trace on $\partial \Omega$.
} 
Remark 5.5. The previous argument can be easily adapted to give a shorter proof of the mountain pass characterization of the second Dirichlet eigenvalue of the $p$-Laplacian (see [11, Theorem 3.1]).

In what follows, we will use the shortcut notation

$$
\mathcal{R}_{\Omega}(u)=\frac{\int_{\Omega}\|\nabla u\|_{\ell^{p}}^{p} d x}{\int_{\partial \Omega}|u|^{p} \varrho d \mathcal{H}^{N-1}}, \quad u \in W^{1, p}(\Omega) \backslash\{0\},
$$

where it is understood that $\mathcal{R}_{\Omega}(u)=+\infty$ whenever $u$ has zero trace on the boundary. The following is the main result of this section. It gives a simpler variational description of $\sigma_{2, p}(\Omega)$ just in terms of a constrained minimization, rather than through a minimax procedure.

Theorem 5.6. Let $\Omega \subset \mathbb{R}^{N}$ be an open bounded Lipschitz set and $\varrho: \partial \Omega \rightarrow \mathbb{R}$ be a function such that (3.1) holds. Then the infimum

$$
\inf _{u \in W^{1, p}(\Omega) \backslash\{0\}}\left\{\mathcal{R}_{\Omega}(u): \int_{\partial \Omega}|u|^{p-2} u \varrho d \mathcal{H}^{N-1}=0\right\},
$$

is attained and coincides with $\sigma_{2, p}(\Omega)$. Moreover, every minimizer of $(5.5)$ is a Stekloff eigenfunction.

Proof. We first observe that if $\Omega$ is not connected, then the infimum in (5.5) is 0 . Since in this case we have $\sigma_{2, p}(\Omega)=0$ as well (see Remark 4.3), then the result is proven.

Let us now suppose that $\Omega$ is connected. We start by proving that the infimum (5.5) is attained. A standard contradiction argument (see [22, Theorem 3.20] for example) exploiting the compactness of the embedding $W^{1, p}(\Omega) \hookrightarrow$ $L^{p}(\partial \Omega)$ leads to the existence of a constant $C_{p, \Omega}$ such that

$$
\int_{\partial \Omega}|v|^{p} \varrho d \mathcal{H}^{N-1} \leq C_{p, \Omega} \int_{\Omega}|\nabla v|^{p} d x
$$

for all $v \in W^{1, p}(\Omega)$ verifying

$$
\int_{\partial \Omega}|v|^{p-2} v \varrho d \mathcal{H}^{N-1}=0 .
$$

Then, by the equivalence of all norms in $\mathbb{R}^{N}$, it is not difficult to deduce that

$$
\mathcal{R}_{\Omega}(u) \geq C_{p, \Omega}>0, \quad \text { for all } \varphi \in W^{1, p}(\Omega) \text { satisfying (5.6), }
$$

possibly for a different constant $C_{p, \Omega}$. This shows that the infimum (5.5) is strictly positive. The existence of a minimizer is again a straightforward consequence of Lemma 2.1 and of the compact embedding $W^{1, p}(\Omega) \hookrightarrow L^{p}(\partial \Omega)$.

We now denote by $\sigma^{*}$ the minimum value (5.5) and take a function $u \in$ $W^{1, p}(\Omega)$ realizing it. Then $u$ minimizes the functional

$$
v \mapsto \int_{\Omega}\|\nabla v\|_{\ell^{p}}^{p} d x-\sigma^{*} \int_{\partial \Omega}|v|^{p} \varrho d \mathcal{H}^{N-1},
$$

as well, among functions $v \in W^{1, p}(\Omega)$ satisfying the zero-mean condition (5.6). The Euler-Lagrange equation corresponding to this problem is precisely given 
by (3.3) with $\sigma=\sigma^{*}$, since the Lagrange multiplier corresponding to (5.6) is zero. We point out that some care is needed for the case $1<p<2$, see Lemma 5.8 below. This in turn implies that $\sigma^{*}$ is a Stekloff eigenvalue.

Let us now suppose that $u \in W^{1, p}(\Omega)$ is an eigenfuction for some eigenvalue $\sigma \neq 0$, then by testing equation (3.3) with $\varphi=u$, we necessarily have $\mathcal{R}_{\Omega}(u)=\sigma$. Similarly, by taking a constant test function in $\varphi$ in (3.3), we get that $u$ verifies (5.6). This implies that each nontrivial Stekloff eigenfunction $u$ is admissible for problem (5.5) and thus

$$
\sigma^{*} \leq \sigma_{2, p}(\Omega) .
$$

By Theorem 5.1, the reverse inequality holds as well, since $\sigma^{*}>0$.

Remark 5.7. The value (5.5) coincides with the best constant in the following Poincaré-Wirtinger trace inequality

$$
c_{\Omega}\left[\min _{t \in \mathbb{R}} \int_{\partial \Omega}|u+t|^{p} \varrho d \mathcal{H}^{N-1}\right] \leq \int_{\Omega}\|\nabla u\|_{\ell^{p}}^{p} d x, \quad u \in W^{1, p}(\Omega) .
$$

It is sufficient to observe that for every $u \in W^{1, p}(\Omega)$, the function $t \mapsto \| u+$ $t \|_{L^{p}(\partial \Omega ; \varrho)}^{p}$ is $C^{1}$ strictly convex and coercive (see (5.7) below), then the value

$$
\min _{t \in \mathbb{R}} \int_{\partial \Omega}|u+t|^{p} \varrho d \mathcal{H}^{N-1},
$$

is uniquely attained and we have

$$
t \text { minimizes } \int_{\partial \Omega}|u+t|^{p} \varrho d \mathcal{H}^{N-1} \Longleftrightarrow u+t \text { is admissible in (5.5). }
$$

We conclude this section with the following technical result, which we used to deduce the characterization of $\sigma_{2, p}$ given by Theorem 5.6.

Lemma 5.8. (Euler-Lagrange equation) Let $\Omega \subset \mathbb{R}^{N}$ be an open bounded set, having Lipschitz boundary. Let $u \in W^{1, p}(\Omega)$ be a minimizer of the functional

$$
\mathfrak{F}_{p}(v)=\frac{1}{p} \int_{\Omega}\|\nabla v\|_{\ell^{p}}^{p} d x-\frac{\sigma}{p} \int_{\partial \Omega}|v|^{p} \varrho d \mathcal{H}^{N-1}, \quad v \in W^{1, p}(\Omega),
$$

on the set of admissible functions $\mathcal{A}=\left\{v \in W^{1, p}(\Omega): \int_{\partial \Omega}|v|^{p-2} v \varrho d \mathcal{H}^{N-1}=0\right\}$. Then $u$ is a Stekloff eigenfunction of $\widetilde{\Delta}_{p}$ with eigenvalue $\sigma$.

Proof. For $p \geq 2$, observe that $\mathcal{A}$ is a $C^{1}$ manifold, thus the thesis is a plain consequence of the Lagrange Multipliers Theorem. Indeed, in this case $u$ has to satisfy

$$
\begin{aligned}
& \sum_{i=1}^{N} \int_{\Omega}\left|u_{x_{i}}\right|^{p-2} u_{x_{i}} \varphi_{x_{i}} d x-\sigma \int_{\partial \Omega}|u|^{p-2} u \varphi \varrho d \mathcal{H}^{N-1} \\
& \quad+\mu \int_{\Omega}|u|^{p-2} \varphi \varrho d \mathcal{H}^{N-1}=0, \quad \text { for every } \varphi \in W^{1, p}(\Omega),
\end{aligned}
$$

for some $\mu \in \mathbb{R}$. By choosing as $\varphi$ any constant function and by using that $u \in \mathcal{A}$, we can then easily conclude that $\mu=0$, i.e. $u$ satisfies (3.3). 
For $1<p<2$ some care is needed, since the constraint $\mathcal{A}$ is no more a $C^{1}$ manifold and we can not directly conclude as before. In this case, we adapt the argument in [13]: observe that we are not assuming $u$ to be in $L^{\infty}(\Omega)$. Let $\varphi \in \operatorname{Lip}(\Omega)$ and $n \in \mathbb{N} \backslash\{0\}$, then the $C^{1}$ convex function

$$
h_{n}(c)=\int_{\partial \Omega}\left|u+\frac{1}{n} \varphi+c\right|^{p} \varrho d \mathcal{H}^{N-1}, \quad c \in \mathbb{R},
$$

is coercive, since we have

$$
h_{n}(c) \geq 2^{1-p}|c|^{p} \int_{\partial \Omega} \varrho d \mathcal{H}^{N-1}-\int_{\partial \Omega}\left|u+\frac{1}{n} \varphi\right|^{p} \varrho d \mathcal{H}^{N-1} .
$$

In particular, for every $n \in \mathbb{N} \backslash\{0\}, h_{n}$ admits a minimum point $c_{n}$, which thus satisfies $h_{n}^{\prime}\left(c_{n}\right)=0$, that is

$$
\int_{\partial \Omega}\left|u+\frac{1}{n} \varphi+c_{n}\right|^{p-2}\left(u+\frac{1}{n} \varphi+c_{n}\right) \varrho d \mathcal{H}^{N-1}=0,
$$

i.e. $u+1 / n \varphi+c_{n} \in \mathcal{A}$. Moreover, we can guarantee that there exists a constant $M$ such that

$$
\left|n c_{n}\right| \leq M, \quad \text { for every } n \in \mathbb{N} .
$$

Indeed, if this would not be true, then there would exist a subsequence $\left\{n_{k} c_{n_{k}}\right\}_{k \in \mathbb{N}}$ such that $n_{k} c_{n_{k}} \rightarrow \infty$ or $n_{k} c_{n_{k}} \rightarrow-\infty$. Since $\varphi$ is bounded on $\partial \Omega$, then for every $k \in \mathbb{N}$ sufficiently large we would have either

$\varphi(x)+n_{k} c_{n_{k}}>0$ for every $x \in \partial \Omega$ or $\varphi(x)+n_{k} c_{n_{k}}<0$ for every $x \in \partial \Omega$.

By observing that the function $\tau \mapsto|u+\tau|^{p-2} \tau$ is strictly increasing, we would obtain

$$
\begin{aligned}
0 & =\int_{\partial \Omega}\left|u+\frac{1}{n_{k}} \varphi+c_{n_{k}}\right|^{p-2}\left(u+\frac{1}{n_{k}} \varphi+c_{n_{k}}\right) \varrho d \mathcal{H}^{N-1} \\
& >\int_{\partial \Omega}|u|^{p-2} u \varrho d \mathcal{H}^{N-1}=0,
\end{aligned}
$$

or

$$
\begin{aligned}
0= & \int_{\partial \Omega}\left|u+\frac{1}{n_{k}} \varphi+c_{n_{k}}\right|^{p-2}\left(u+\frac{1}{n_{k}} \varphi+c_{n_{k}}\right) \varrho d \mathcal{H}^{N-1} \\
& <\int_{\partial \Omega}|u|^{p-2} u \varrho d \mathcal{H}^{N-1}=0 .
\end{aligned}
$$

In both cases we would get a contradiction, so (5.8) must be true. This in turn implies that, possibly by passing to a subsequence, the sequence $\left\{n c_{n}\right\}_{n \in \mathbb{N}}$ converges to some real number $C$, as $n$ goes to $\infty$. By using the minimality of $u$ and the fact that $u+1 / n \varphi+c_{n}$ is admissible, we then get 


$$
\begin{aligned}
0 \leq \lim _{n \rightarrow \infty} \frac{\mathfrak{F}_{p}\left(u+\frac{1}{n}\left(\varphi+n c_{n}\right)\right)-\mathfrak{F}_{p}(u)}{\frac{1}{n}}= & \sum_{i=1}^{N} \int_{\Omega}\left|u_{x_{i}}\right|^{p-2} u_{x_{i}} \varphi_{x_{i}} d x \\
& -\sigma \int_{\partial \Omega}|u|^{p-2} u(\varphi+C) \varrho d \mathcal{H}^{N-1} .
\end{aligned}
$$

Since $u \in \mathcal{A}$, the previous is equivalent to

$$
0 \leq \sum_{i=1}^{N} \int_{\Omega}\left|u_{x_{i}}\right|^{p-2} u_{x_{i}} \varphi_{x_{i}} d x-\sigma \int_{\partial \Omega}|u|^{p-2} u \varphi \varrho d \mathcal{H}^{N-1}, \quad \varphi \in \operatorname{Lip}(\Omega) .
$$

The same argument with $-\varphi$ in place of $\varphi$ shows that $u$ satisfies equation (3.3), for every Lipschitz test function. The conclusion then follows by exploiting the density of Lipschitz functions in $W^{1, p}(\Omega)$, which is true since $\Omega$ has Lipschitz boundary (see [22, Theorem 3.6]).

\section{Further properties}

The next result concerns some nodal properties of the first nontrivial eigenvalue. The proof is inspired to the linear case (see $[2,25])$.

Proposition 6.1. Let $\Omega \subset \mathbb{R}^{N}$ be an open and connected bounded set, having Lipschitz boundary. There exists a first nontrivial Stekloff eigenfunction $w \in W^{1, p}(\Omega)$ with exactly two nodal domains.

Proof. Let us take $u \in W^{1, p}(\Omega)$ a first nontrivial eigenfuction, thanks to Lemma 3.5 we have that $u$ has at least two nodal domains.

Let us now suppose that $u$ has $n \geq 3$ nodal domains, $\Omega_{1}, \ldots, \Omega_{n} \subset \Omega$. We then take the functions

$$
v_{k}=u \cdot 1_{\Omega_{k}}, \quad k=1,2,
$$

i.e. the restrictions of $u$ to $\Omega_{1}$ and $\Omega_{2}$, respectively and we define

$$
w=\alpha v_{1}+\beta v_{2} \text {. }
$$

This is a function in $W^{1, p}(\Omega)$ and observe that we can always choose $\alpha, \beta \in$ $\mathbb{R} \backslash\{0\}$ such that

$$
\int_{\partial \Omega}|w|^{p-2} w \varrho d \mathcal{H}^{N-1}=0 .
$$

By construction $w$ is admissible for the variational problem (5.5) which gives $\sigma_{2, p}(\Omega)$. Moreover, we can infer

$$
\begin{aligned}
\int_{\Omega}\|\nabla w\|_{\ell^{p}}^{p} d x & =\alpha^{p} \int_{\Omega_{1}}\left\|\nabla v_{1}\right\|^{p} d x+\beta^{p} \int_{\Omega_{2}}\left\|\nabla v_{2}\right\|^{p} d x \\
& =\sigma_{2, p}(\Omega)\left[\alpha^{p} \int_{\partial \Omega_{1} \cap \partial \Omega}\left|v_{1}\right|^{p} \varrho d \mathcal{H}^{N-1}+\beta^{p} \int_{\partial \Omega_{2} \cap \partial \Omega}\left|v_{2}\right|^{p} \varrho d \mathcal{H}^{N-1}\right] \\
& =\sigma_{2, p}(\Omega) \int_{\partial \Omega}|w|^{p} \varrho d \mathcal{H}^{N-1} .
\end{aligned}
$$


Owing to the characterization of Theorem 5.6 for $\sigma_{2, p}(\Omega)$, we then get that $w$ is a first nontrivial Stekloff eigenfuction of $\Omega$, having exactly two nodal domains.

Remark 6.2. Very likely the previous property is verified by every Stekloff eigenfunction corresponding to $\sigma_{2, p}(\Omega)$, i.e. every first nontrivial eigenfunction should have exactly two nodal domains. The main obstruction to the proof of this fact is the lack of a unique continuation principle for pseudo $p$-harmonics functions. Indeed, observe that in the previous proof we constructed a function $w$ which satisfies $\widetilde{\Delta} w=0$ and identically vanishes on a open subset of $\Omega$, but we can not get a contradiction from this. We also like to point out that Harnack's inequality is of not use here, since we can not guarantee that $\partial \Omega_{1} \cap \Omega$ does not coincide with $\partial \Omega_{2} \cap \Omega$. This is linked to the existence of the so-called Lakes of Wada, i.e. triples of open connected sets in the plane, which share the same boundaries.

In the case of the second Dirichlet eigenvalue of the $p$-Laplacian, the use of the unique continuation property can be avoided, as proved in [12]. However, also this proof can not be applied here, since our eigenfunctions are not known to be in $C^{1}$, as required by the argument in [12].

Definition 6.3. Let $\Omega \subset \mathbb{R}^{N}$ be an open bounded connected set, with Lipschitz boundary. Let us consider two open connected Lipschitz subsets $\Omega_{1}, \Omega_{2} \subset \Omega$, then $\left(\Omega_{1}, \Omega_{2}\right)$ is said a halving pair for $\Omega$ if the following conditions are satisfied:

$$
\Omega_{1} \cap \Omega_{2}=\emptyset \quad \text { and } \quad \mathcal{H}^{N-1}\left(\partial \Omega_{i} \cap \partial \Omega\right)>0, \quad i=1,2 .
$$

We also set

$$
\operatorname{Hal}(\Omega)=\left\{\left(\Omega_{1}, \Omega_{2}\right) \text { halving pair of } \Omega\right\} \text {. }
$$

If $\Sigma \subset \Omega$ is such that $\Gamma:=\partial \Sigma \cap \Omega \neq \emptyset$ and this is a Lipschitz hypersurface, we also introduce the following quantity

$$
\Lambda_{p}(\Sigma ; \Omega)=\min _{u \in W^{1, p}(\Sigma) \backslash\{0\}}\left\{\mathcal{R}_{\Sigma}(u): u=0 \text { on } \Gamma\right\},
$$

where $\mathcal{R}_{\Sigma}$ is still defined by (5.4). An optimal function in (6.2) is a weak solution of the following mixed Dirichlet-Stekloff eigenvalue problem

$$
\begin{cases}\widetilde{\Delta}_{p} u=0, & \text { in } \Sigma \\ u=0, & \text { on } \Gamma, \\ \sum_{i=1}^{N}\left|u_{x_{i}}\right|^{p-2} u_{x_{i}} \nu_{\Omega}^{i}=\lambda|u|^{p-2} u \varrho, & \text { on } \partial \Omega \cap \partial \Sigma,\end{cases}
$$

with $\lambda=\Lambda_{p}(\Sigma ; \Omega)$, i.e. a minimizer of (6.2) satisfies

$$
\sum_{i=1}^{N} \int_{\Omega}\left|u_{x_{i}}\right|^{p-2} u_{x_{i}} \varphi_{x_{i}} d x=\Lambda_{p}(\Sigma ; \Omega) \int_{\partial \Omega \cap \partial \Sigma}|u|^{p-2} u \varphi \varrho d \mathcal{H}^{N-1}
$$

for every $\varphi \in W^{1, p}(\Sigma)$ with $\varphi=0$ on $\Gamma$. 
Lemma 6.4. With the previous notation, for every $p \in(1, \infty)$ problem $(6.2)$ admits a unique positive solution $u \in W^{1, p}(\Sigma)$ satisfying the normalization condition

$$
\int_{\partial \Omega \cap \partial \Sigma}|u|^{p} \varrho d \mathcal{H}^{N-1}=1
$$

Moreover, the boundary value problem (6.3) admits a positive (weak) solution if and only if $\lambda=\Lambda_{p}$.

Proof. Existence of a solution for this problem is straightforward. Positivity follows as always by observing that for every admissible $u$, the function $|u|$ is still admissible and

$$
\mathcal{R}_{\Sigma}(|u|)=\mathcal{R}_{\Sigma}(u)
$$

Uniqueness can be proved using the convexity argument, that we already used in Lemma 5.2. Suppose to have two distinct strictly positive ${ }^{5}$ solutions $u_{0}$ and $u_{1}$ such that

$$
\int_{\partial \Omega \cap \partial \Sigma}\left|u_{i}\right|^{p} \varrho d \mathcal{H}^{N-1}=1, \quad i=0,1 .
$$

As in Lemma 5.2, we set $\gamma_{t}(x)=\left[(1-t) u_{0}(x)^{p}+t u_{1}(x)^{p}\right]^{1 / p}$, for a given $0<t<1$. This still satisfies the normalization condition (6.4) and

$$
t \mapsto \mathcal{R}_{\Sigma}\left(\gamma_{t}\right) \text { is strictly convex on }[0,1] .
$$

Then $\gamma_{t}$ is still a solution and we must have

$$
\mathcal{R}_{\Sigma}\left(\gamma_{t}\right)=\mathcal{R}_{\Sigma}\left(u_{0}\right)=\mathcal{R}_{\Sigma}\left(u_{1}\right), \quad t \in[0,1] .
$$

This can hold if and only if $u_{0}=\mu u_{1}$ for some $\mu>0$ (see [5] for more details). By using (6.4), we get $\mu=1$ and thus we obtain a contradiction.

The second part of the statement can be proved along the same lines of [8, Theorem 3.1], still using property (6.5). One just needs to observe that every $\lambda$ such that (6.3) has a solution is a crititical value of $\int_{\Omega}\|\nabla u\|_{\ell^{p}}^{p}$ on the manifold

$$
\left\{v \in W^{1, p}(\Omega): v=0 \text { on } \Gamma \quad \text { and } \quad \int_{\partial \Omega \cap \partial \Sigma}|v|^{p} \varrho d \mathcal{H}^{N-1}=1\right\} .
$$

This concludes the proof.

By using problem (6.3), we have yet another minimax characterization of $\sigma_{2, p}(\Omega)$, this time in terms of the eigenvalues $\Lambda_{p}$. For this, we need to assume some smoothness on the nodal domains.

\footnotetext{
${ }^{5}$ Strict positivity is a consequence of Harnack's inequality. Indeed, as already observed, a pseudo $p$-harmonic function is a local minimizer of the Dirichlet energy $\int_{\Omega}\|\nabla u\|_{\ell^{p}}^{p} d x$. Then Harnack's inequality for these functions is a consequence of [22, Theorem 7.11].
} 
Proposition 6.5. Let $\Omega \subset \mathbb{R}^{N}$ be an open bounded connected set, having Lipschitz boundary. Suppose that the nodal domains $\Omega_{+}$and $\Omega_{-}$of the first nontrivial eigenfunction $w$ constructed in Proposition 6.1 belong to $\operatorname{Hal}(\Omega)$. Then there holds

$$
\sigma_{2, p}(\Omega)=\min \left\{\max \left\{\Lambda_{p}\left(\Omega_{1} ; \Omega\right), \Lambda_{p}\left(\Omega_{2} ; \Omega\right)\right\}:\left(\Omega_{1}, \Omega_{2}\right) \in \operatorname{Hal}(\Omega)\right\} .
$$

The minimum above is realized by the pair $\left(\Omega_{+}, \Omega_{-}\right)$and

$$
\Lambda_{p}\left(\Omega_{+} ; \Omega\right)=\mathcal{R}_{\Omega_{+}}(w)=\mathcal{R}_{\Omega_{-}}(w)=\Lambda_{p}\left(\Omega_{-} ; \Omega\right) .
$$

Proof. Let us take a halving pair $\left(\Omega_{1}, \Omega_{2}\right)$ and $u_{i} \in W^{1, p}\left(\Omega_{i}\right)$ such that $u_{i}=0$ on $\partial \Omega_{i} \cap \Omega$, with

$$
\int_{\Omega_{i}}\left\|\nabla u_{i}\right\|_{\ell^{p}}^{p} d x=\Lambda_{p}\left(\Omega_{i} ; \Omega\right) \quad \text { and } \quad \int_{\partial \Omega_{i} \cap \partial \Omega}\left|u_{i}\right|^{p} \varrho d \mathcal{H}^{N-1}=1, \quad i=1,2 .
$$

Then we can choose two parameters $\alpha_{1}, \alpha_{2} \in \mathbb{R}$ in such a way that

$$
v(x)=\sum_{i=1}^{2} \alpha_{i} u_{i}(x) \cdot 1_{\Omega_{i}}(x), \quad x \in \Omega,
$$

satisfies the zero-mean condition (5.6). Thus, we can infer

$$
\begin{aligned}
\sigma_{2, p}(\Omega) & \leq \frac{\alpha_{1}^{p} \int_{\Omega_{1}}\left\|\nabla u_{1}\right\|_{\ell^{p}}^{p} d x+\alpha_{2}^{p} \int_{\Omega_{2}}\left\|\nabla u_{2}\right\|_{\ell^{p}}^{p} d x}{\alpha_{1}^{p}+\alpha_{2}^{p}} \\
& =\frac{\alpha_{1}^{p} \Lambda_{p}\left(\Omega_{1} ; \Omega\right)+\alpha_{2}^{p} \Lambda_{p}\left(\Omega_{2} ; \Omega\right)}{\alpha_{1}^{p}+\alpha_{2}^{p}} \leq \max \left\{\Lambda_{p}\left(\Omega_{1} ; \Omega\right), \Lambda_{p}\left(\Omega_{2} ; \Omega\right)\right\},
\end{aligned}
$$

and since this is true for every halving pair $\left(\Omega_{1}, \Omega_{2}\right)$, this remains true taking the infimum over $\operatorname{Hal}(\Omega)$.

Let us now take the eigenfunction $w \in W^{1, p}(\Omega)$ relative to $\sigma_{2, p}(\Omega)$, constructed in Proposition 6.1. Its two nodal domains $\Omega_{+}$and $\Omega_{-}$both touch the boundary of $\Omega$, by Lemma 3.5. Using the equation, we then have

$$
\mathcal{R}_{\Omega_{+}}(w)=\mathcal{R}_{\Omega_{-}}(w)=\sigma_{2, p}(\Omega) .
$$

By definition of $\Lambda_{p}$ and the hypothesis on $\Omega_{+}, \Omega_{-}$, we then get

$$
\Lambda_{p}\left(\Omega_{+} ; \Omega\right) \leq \mathcal{R}_{\Omega_{+}}(w) \text { and } \Lambda_{p}\left(\Omega_{-} ; \Omega\right) \leq \mathcal{R}_{\Omega_{-}}(w)
$$

so that

$$
\max \left\{\Lambda_{p}\left(\Omega_{+} ; \Omega\right), \Lambda_{p}\left(\Omega_{-} ; \Omega\right)\right\} \leq \sigma_{2, p}(\Omega) .
$$

This concludes the proof of (6.6) and shows that the minimum is realized by the pair $\left(\Omega_{+}, \Omega_{-}\right)$. In order to prove $(6.7)$, it is sufficient to observe that $w$ restricted to $\Omega_{+}$is a positive solution of $(6.3)$, with $\lambda=\sigma_{2, p}(\Omega)$. By the second part of Lemma 6.4 , we can infer that $\Lambda_{p}\left(\Omega_{+} ; \Omega\right)=\mathcal{R}_{\Omega_{+}}(w)$. The same observation applies to $\Omega_{-}$, thus leading to $(6.7)$. 


\section{An upper bound for $\sigma_{2, p}$}

In this section we prove an upper bound for $\sigma_{2, p}(\Omega)$, in terms of geometric quantities. For this, we need the following simple result. It guarantees that the coordinate functions $\varphi_{j}(x)=x_{j}, j=1, \ldots, N$ are always admissible in (5.5), modulo a translation.

Lemma 7.1. Let $\Omega \subset \mathbb{R}^{N}$ be a bounded open set, having Lipschitz boundary. Let $\varrho: \partial \Omega \rightarrow \mathbb{R}$ be a function satisfying (3.1). Then there exists $z \in \mathbb{R}^{N}$ such that the translated set $\Omega^{\prime}=\Omega-z$ satisfies

$$
\int_{\partial \Omega^{\prime}}\left|x_{i}\right|^{p-2} x_{i} \varrho(x+z) d \mathcal{H}^{N-1}(x)=0, \quad i=1, \ldots, N .
$$

Proof. Let us consider the following function

$$
g(y)=\sum_{i=1}^{N} \frac{1}{p} \int_{\partial \Omega}\left|x_{i}-y_{i}\right|^{p} \varrho(x) d \mathcal{H}^{N-1}(x), \quad y=\left(y_{1}, \ldots, y_{N}\right) \in \Omega
$$

It is not difficult to see that this function is $C^{1}$ and admits a global minimum point, thus there exists $z$ such that

$$
\int_{\partial \Omega}\left|x_{i}-z_{i}\right|^{p-2}\left(x_{i}-z_{i}\right) \varrho(x) d \mathcal{H}^{N-1}(x)=0, \quad i=1, \ldots, N .
$$

Let us now make the change of variable $y=x-z$. By defining $\Omega^{\prime}=\Omega-z$, the previous turns out to be equal to

$$
\int_{\partial \Omega^{\prime}}\left|y_{i}\right|^{p-2} y_{i} \varrho(y+z) d \mathcal{H}^{N-1}(y)=0, \quad i=1, \ldots, N
$$

which gives the thesis.

The following is the main result of this section, dealing with the case of a general weight $\varrho$ satisfying (3.1). This is the nonlinear counterpart of Brock's inequality for the first nontrivial Stekloff eigenvalue of the Laplacian (compare with [9, Theorem 1]). Its proof crucially exploits the weighted Wulff inequality derived in Theorem A.4 and Corollary A.6 of the Appendix.

Theorem 7.2. Let $1<p<\infty$ and $p^{\prime}=p /(p-1)$. Let $\Omega \subset \mathbb{R}^{N}$ be an open bounded set, having Lipschitz boundary and $\varrho$ a function satisfying (3.1). Then there holds

$$
\sigma_{2, p}(\Omega) \leq\left(\frac{\int_{\partial \Omega} \varrho^{-\frac{1}{p-1}}\left\|\nu_{\Omega}\right\|_{\ell^{p^{\prime}}}^{p^{\prime}} d \mathcal{H}^{N-1}}{N|\Omega|}\right)^{p-1}
$$

Proof. Let $z \in \mathbb{R}^{N}$ be as in Lemma 7.1 and let us set $\Omega^{\prime}=\Omega-z$. By the characterization (5.5) of $\sigma_{2, p}(\Omega)$, we obtain 


$$
\begin{aligned}
\sigma_{2, p}(\Omega) & \leq \frac{\int_{\Omega^{\prime}}\left\|\nabla \varphi_{i}\right\|_{\ell^{p}}^{p} d x}{\int_{\partial \Omega^{\prime}}\left|\varphi_{i}(x)\right|^{p} \varrho(x+z) d \mathcal{H}^{N-1}(x)} \\
& =\frac{|\Omega|}{\int_{\partial \Omega^{\prime}}\left|x_{i}\right|^{p} \varrho(x+z) d \mathcal{H}^{N-1}(x)}, \quad i=1, \ldots, N,
\end{aligned}
$$

where $\varphi_{i}(x)=x_{i}$, as before. By taking the sum over $i=1, \ldots, N$, we obtain

$$
\sigma_{2, p}(\Omega) \leq \frac{N|\Omega|}{\int_{\partial \Omega^{\prime}}\|x\|_{\ell^{p}}^{p} \varrho(x+z) d \mathcal{H}^{N-1}(x)} .
$$

Then we observe that by Hölder inequality we have

$\int_{\partial \Omega^{\prime}}\|x\|_{\ell^{p}}^{p} \varrho(x+z) d \mathcal{H}^{N-1}(x) \geq \frac{\left(\int_{\partial \Omega^{\prime}}\|x\|_{\ell^{p}}\left\|\nu_{\Omega^{\prime}}(x)\right\|_{\ell^{p^{\prime}}} d \mathcal{H}^{N-1}(x)\right)^{p}}{\left(\int_{\partial \Omega^{\prime}} \varrho(x+z)^{-\frac{1}{p-1}}\left\|\nu_{\Omega^{\prime}}(x)\right\|_{\ell^{p^{\prime}}}^{p^{\prime}} d \mathcal{H}^{N-1}(x)\right)^{p-1},}$

and the numerator in the right-hand side is the weigthed anisotropic perimeter $P_{p, 1}\left(\Omega^{\prime}\right)$ of $\Omega^{\prime}$, with the notation (A.13). Also observe that $\nu_{\Omega^{\prime}}(x)=\nu_{\Omega}(x+z)$, then using the weigthed Wulff inequality of Corollary A.6 with $\beta=1$, we get

$$
\begin{aligned}
\sigma_{2, p}(\Omega) & \leq \frac{N|\Omega|}{\left(\int_{\partial \Omega^{\prime}}\|x\|_{\ell p}\left\|\nu_{\Omega^{\prime}}(x)\right\|_{\ell^{p^{\prime}}} d \mathcal{H}^{N-1}(x)\right)^{p}}\left(\int_{\partial \Omega} \varrho(x)^{-\frac{1}{p-1}}\left\|\nu_{\Omega}(x)\right\|_{\ell^{p^{\prime}}}^{p^{\prime}} d \mathcal{H}^{N-1}(x)\right)^{p-1} \\
& \leq \frac{N|\Omega|}{N^{p}|\Omega|^{p}}\left(\int_{\partial \Omega} \varrho(x)^{-\frac{1}{p-1}}\left\|\nu_{\Omega}(x)\right\|_{\ell^{p^{\prime}}}^{p^{\prime}} d \mathcal{H}^{N-1}(x)\right)^{p-1}
\end{aligned}
$$

which gives the desired estimate.

A significant and more intrinsic instance of weight function $\varrho$ verifying (3.1) is given by

$$
\varrho(x)=\left\|\nu_{\Omega}(x)\right\|_{\ell^{p^{\prime}}}, \quad x \in \partial \Omega .
$$

In this case, an elegant and simpler bound is possible, that should be compared with the Brock-Weinstock inequality (1.4).

Theorem 7.3. Let $\Omega \subset \mathbb{R}^{N}$ be an open bounded set, having Lipschitz boundary. Let us still indicate by $\sigma_{2, p}(\Omega)$ the Stekloff eigenvalue corresponding to the choice (7.3) for the weight $\varrho$. Then there holds

$$
\sigma_{2, p}(\Omega) \leq\left(\frac{\left|B_{p}\right|}{|\Omega|}\right)^{\frac{p-1}{N}},
$$

where $B_{p}=\left\{x \in \mathbb{R}^{N}:\|x\|_{\ell^{p}}<1\right\}$.

Proof. Again, we take $\varphi_{i}(x)=x_{i}, i=1, \ldots, N$, then up to a translation of $\Omega$ (which does not affect $\sigma_{2, p}(\Omega)$ ), we can suppose that $(7.1)$ is satisfied. We again obtain 


$$
\sigma_{2, p}(\Omega) \leq \frac{\int_{\Omega}\left\|\nabla \varphi_{i}\right\|_{\ell^{p}}^{p} d x}{\int_{\partial \Omega}\left|\varphi_{i}\right|^{p}\left\|\nu_{\Omega}\right\|_{\ell^{p^{\prime}}} d \mathcal{H}^{N-1}}=\frac{|\Omega|}{\int_{\partial \Omega}\left|x_{i}\right|^{p}\left\|\nu_{\Omega}\right\|_{\ell^{p^{\prime}}} d \mathcal{H}^{N-1}}, \quad i=1, \ldots, N .
$$

That is, summing up over $i=1, \ldots, N$, we have

$$
\sigma_{2, p}(\Omega) \leq \frac{N|\Omega|}{\int_{\partial \Omega}\|x\|_{\ell^{p}}^{p}\left\|\nu_{\Omega}\right\|_{\ell^{p^{\prime}}} d \mathcal{H}^{N-1}} .
$$

Using the isoperimetric property of $B_{p}$ given by Corollary A.6, this time with $\beta=p$, we eventually obtain the thesis.

Remark 7.4. We conjecture the bounds (7.2) and (7.4) to be "isoperimetric", like in the linear case corresponding to the Brock-Weinstock inequality (1.4). In other words, we conjecture that equality holds in (7.4) if and only if $\Omega=B_{p}$, up to dilations and translations. For (7.2) one also needs to require

$$
\varrho(x)=c\left\|\nu_{\Omega}(x)\right\|_{\ell^{p^{\prime}}}, \quad x \in \partial \Omega .
$$

To prove this conjecture, one would need to show that $\sigma_{2, p}\left(B_{p}\right)=1$, i.e. the coordinate functions $\varphi_{i}(x)=x_{i}, i=1, \ldots, N$ are first nontrivial eigenfuctions of $\widetilde{\Delta}_{p}$ on $B_{p}$. It is easily seen that $x_{1}, \ldots, x_{N}$ are indeed Stekloff eigenfunctions on $B_{p}$, with corresponding eigenvalue 1 . Of course, it could happen that $\sigma_{2, p}\left(B_{p}\right)<1$. To conclude, it would be sufficient to prove the existence of a first nontrivial eigenfunction having $\left\{x_{j}=0\right\}$ as nodal line, for some $j=1, \ldots, N$. The thesis would then follows from Lemma 6.4 and Proposition 6.5.

\section{Acknowledgments}

We wish to thank Luigi De Pascale, Guido De Philippis and Carlo Nitsch for some useful conversations. Part of this work has been written during a visit of the second author to Marseille. The FRUMAM and LATP institutions and their facilities are gratefully acknowledged. G. F. has been partially supported by the Ph.D. program of the University of Trento.

\section{Appendix A: Weighted Wulff inequalities}

We start this self-contained Appendix with some facts about the theory of convex bodies. For more details, the reader should consult [29].

Let $K \subset \mathbb{R}^{N}$ be a bounded convex set with non empty interior, containing the origin. Here and in what follows we will always take $N \geq 2$. We define the 1-positively homogeneous function

$$
\|x\|=\inf \{\lambda>0: x \in \lambda K\}
$$

and observe that $K$ turns out to be the unit ball for this "norm" (actually, this is not a true norm since $\|-x\| \neq\|x\|$ ). We also define the dual norm

$$
\|\xi\|_{*}=\max _{x \in K}\langle x, \xi\rangle,
$$


which is sometimes called support function of $K$. Then the polar set $K^{*}$ is usually defined as the unit ball for $\|\cdot\|_{*}$, i.e.

$$
K^{*}=\left\{\xi \in \mathbb{R}^{N}:\|\xi\|_{*} \leq 1\right\} .
$$

By definition, we have the following general version of the Cauchy-Schwarz inequality

$$
|\langle x, \xi\rangle| \leq\|x\|\|\xi\|_{*}, \quad x, \xi \in \mathbb{R}^{N} .
$$

If $x \neq 0$, equality holds if and only if $\xi$ belongs to the normal cone $N_{K}(x /\|x\|)$ to $K$ at the point $x /\|x\|$. In particular, if $K$ is $C^{1}$ equality holds if and only if $\xi=t \nu_{K}(x /\|x\|)$, for some $t \geq 0$.

Given $\Omega \subset \mathbb{R}^{N}$ an open bounded Lipschitz set, if we define its anisotropic perimeter by

$$
P_{K}(\Omega)=\int_{\partial \Omega}\left\|\nu_{\Omega}\right\|_{*} d \mathcal{H}^{N-1},
$$

we have the classical Wulff inequality

$$
P_{K}(\Omega) \geq N|K|^{\frac{1}{N}}|\Omega|^{\frac{N-1}{N}} .
$$

By recalling that $P_{K}(K)=N|K|$, the previous is equivalent to say that $K$ minimizes $P_{K}$, among sets with given measure. Moreover, strict equality holds in (A.2), if $\Omega$ is not a scaled and translated copy of $K$. See for example [16] for a detailed study of the Wulff inequality.

Definition A.1. Let $V:[0,+\infty) \rightarrow[0,+\infty)$ be a Borel function such that $V(0)=0$. For every $\Omega \subset \mathbb{R}^{N}$ open bounded Lipschitz set, we define its weighted anisotropic perimeter by

$$
P_{V, K}(\Omega)=\int_{\partial \Omega} V(\|x\|)\left\|\nu_{\Omega}(x)\right\|_{*} d \mathcal{H}^{N-1}(x) .
$$

Remark A.2. When $K$ coincides with the unit ball of the Euclidean norm $|\cdot|$, it easily seen that $\|x\|=\|x\|_{*}=|x|$ and $P_{V, K}$ coincides with the weighted perimeter

$$
\int_{\partial \Omega} V(|x|) d \mathcal{H}^{N-1}(x)
$$

already studied in [6, 7].

Let us now further suppose that $V \in C^{1}([0, \infty))$ and it satisfies the following conditions:

$$
V(t)>0, \quad \text { for } t>0
$$

and

$$
v(t):=V^{\prime}(t)+(N-1) \frac{V(t)}{t}, \quad \text { is non decreasing for } t>0 .
$$

We consider the vector field

$$
W(x)=V(\|x\|) \frac{x}{\|x\|}, \quad x \in \mathbb{R}^{N},
$$


with the convention that $W(0)=0$. The crucial property of $W$ is expressed by the following Lemma, which extends to the anisotropic case a straightforward calculation of the Euclidean one.

Lemma A.3. With the previous notations, there holds

$$
\operatorname{div} W(x)=v(\|x\|), \quad x \in \mathbb{R}^{N} \backslash\{0\} .
$$

In particular, $\operatorname{div} W$ is a non decreasing function of $\|\cdot\|$.

Proof. First of all, we observe that $x \mapsto\|x\|$ is convex and thus differentiable almost everywhere. We also have

$$
\nabla\|x\|=\frac{\nu_{K}\left(\frac{x}{\|x\|}\right)}{\left\|\nu_{K}\left(\frac{x}{\|x\|}\right)\right\|_{*}} \text { and }\left\langle\nu_{K}\left(\frac{x}{\|x\|}\right), x\right\rangle=\left\|\nu_{K}\left(\frac{x}{\|x\|}\right)\right\|_{*}\|x\|,
$$

where these relations hold almost everywhere. Observe that (A.5) is a simple consequence of (A.6). Indeed, by using these we get

$$
\begin{aligned}
\operatorname{div} W(x) & =V^{\prime}(\|x\|)\left\langle\nabla\|x\|, \frac{x}{\|x\|}\right\rangle+N \frac{V(\|x\|)}{\|x\|}-V(\|x\|) \frac{\langle\nabla\|x\|, x\rangle}{\|x\|^{2}} \\
& =V^{\prime}(\|x\|)+(N-1) \frac{V(\|x\|)}{\|x\|}=v(\|x\|), \quad \text { for a.e. } x \in \mathbb{R}^{N},
\end{aligned}
$$

which gives the desired result.

So, let us now prove (A.6): we first recall some basic facts of convex analysis. If $F: \mathbb{R}^{N} \rightarrow \mathbb{R} \cup\{+\infty\}$ is a convex lower semicontinuous function, we have

$$
\xi \in \partial F(x) \text { if and only if } F(x)+F^{*}(\xi)=\langle x, \xi\rangle,
$$

where $F^{*}$ denotes the Legendre-Fenchel conjugate of $F$ and $\partial F(x)$ is the subdifferential of $F$ at the point $x$.

Choosing $F(x)=\|x\|$, it is easy to see that its Legendre-Fenchel conjugate function is given by $F^{*}(\xi)=\delta_{K^{*}}(\xi)$, i.e. the indicator function of the polar set $K^{*}$. This yields

$$
\xi \in \partial\|x\| \quad \text { if and only if } \quad\|\xi\|_{*} \leq 1 \text { and }\langle\xi, x\rangle=\|x\| .
$$

In particular, if $x \neq 0$ and $\xi \in \partial\|x\|$, by (A.1) we get

$$
\|x\|=\langle\xi, x\rangle \leq\|x\|\|\xi\|_{*} \leq\|x\|,
$$

i.e. $\|\xi\|_{*}=1$ and equality holds in (A.1). This implies that if $x \neq 0$, the subdifferential of $\partial\|x\|$ is characterized by

$$
\xi \in \partial\|x\| \quad \text { if and only if } \quad\|\xi\|_{*}=1 \text { and } \xi \in N_{K}\left(\frac{x}{\|x\|}\right) .
$$


Since for almost every $x \in \mathbb{R}^{N}$ we have

$$
\begin{aligned}
\partial\|x\| & =\{\nabla\|x\|\} \quad \text { and } \quad N_{K}\left(\frac{x}{\|x\|}\right) \\
& =\left\{z \in \mathbb{R}^{N}: z=t \nu_{K}\left(\frac{x}{\|x\|}\right) \text { for some } t \geq 0\right\},
\end{aligned}
$$

the characterization (A.7) gives the first relation in (A.6).

Observe that the second relation in (A.6) comes again from the cases of equality in the Cauchy-Schwarz inequality, by simply noticing that

$$
\left\langle\nu_{K}\left(\frac{x}{\|x\|}\right), x\right\rangle=\left\langle\nu_{K}\left(\frac{x}{\|x\|}\right), \frac{x}{\|x\|}\right\rangle\|x\| .
$$

This proves (A.6) and thus the thesis.

We are now ready for the main result of this appendix.

Theorem A.4. (Weighted Wulff inequality) Let $\Omega \subset \mathbb{R}^{N}$ be an open bounded Lipschitz set and $V \in C^{1}([0, \infty))$ a weight function verifying (A.3) and (A.4). Then we have

$$
P_{V, K}(\Omega) \geq N|K|^{\frac{1}{N}}|\Omega|^{1-\frac{1}{N}} V\left(\left(\frac{|\Omega|}{|K|}\right)^{\frac{1}{N}}\right),
$$

with equality if and only if $\Omega$ coincides with $K$, up to dilations. In other words, $K$ is the only minimizer of $P_{K, V}$, under measure constraint, i.e.

$$
P_{V, K}(K)=\min \left\{P_{V, K}(\Omega):|\Omega|=|K|\right\} .
$$

Proof. It is easily seen that (A.8) and (A.9) are equivalent, so let us suppose that $|\Omega|=|K|$. We divide the proof in two steps: first we prove the inequality, then we detect the cases of equality.

Inequality By using the Divergence Theorem and Lemma A.3 we get

$$
\begin{aligned}
\int_{\Omega} v(\|x\|) d x=\int_{\Omega} \operatorname{div} W(x) d x= & \int_{\partial \Omega} V(\|x\|)\left\langle\frac{x}{\|x\|}, \nu_{\Omega}(x)\right\rangle d \mathcal{H}^{N-1}(x) \\
= & \int_{\partial \Omega} V(\|x\|)\left[\left\langle\frac{x}{\|x\|}, \nu_{\Omega}(x)\right\rangle-\left\|\nu_{\Omega}(x)\right\|_{*}\right] d \mathcal{H}^{N-1}(x) \\
& +P_{V, K}(\Omega),
\end{aligned}
$$

while integrating $v$ over $K$ yields

$$
\begin{aligned}
\int_{K} v(\|x\|) d x=\int_{K} \operatorname{div} W(x) d x & =\int_{\partial K} V(\|x\|)\left\langle x, \nu_{K}(x)\right\rangle d \mathcal{H}^{N-1}(x) \\
& =\int_{\partial K} V(\|x\|)\left\|\nu_{\Omega}(x)\right\|_{*} d \mathcal{H}^{N-1}(x)=P_{V, K}(K),
\end{aligned}
$$

since by definition $\|x\|=1$ on $\partial K$. Subtracting the two equalities, we get

$$
P_{V, K}(\Omega)-P_{V, K}(K)=\mathcal{I}_{1}(\Omega)+\mathcal{I}_{2}(\Omega)
$$

where we set

$$
\mathcal{I}_{1}(\Omega)=\int_{\partial \Omega} V(\|x\|)\left[\left\|\nu_{\Omega}(x)\right\|_{*}-\left\langle\frac{x}{\|x\|}, \nu_{\Omega}(x)\right\rangle\right] d \mathcal{H}^{N-1}(x),
$$


and

$$
\mathcal{I}_{2}(\Omega)=\int_{\Omega} v(\|x\|) d x-\int_{K} v(\|x\|) d x .
$$

It is not difficult to see that both quantities are positive. For the first, this is a simple consequence of the Cauchy-Schwarz inequality (A.1); for the second, we just observe that

$$
\mathcal{I}_{2}(\Omega)=\int_{\Omega \backslash K}[v(\|x\|)-v(1)] d x+\int_{K \backslash \Omega}[v(1)-v(\|x\|)] d x
$$

thanks to the fact that $|K \backslash \Omega|=|\Omega \backslash K|$, since $K$ and $\Omega$ have the same measure. On the other hand, there holds

$$
\Omega \backslash K \subset\{x:\|x\| \geq 1\} \text { and } K \backslash \Omega \subset\{x:\|x\| \leq 1\},
$$

then by using the monotone behaviour of $v$, we can infer $\mathcal{I}_{2}(\Omega) \geq 0$. Thus (A.10) shows that $K$ minimizes $P_{V, K}$ among sets with given measure.

Cases of equality Let us suppose that $P_{V, K}(\Omega)=P_{V, K}(K)$. Again by (A.10) we can infer

$$
\mathcal{I}_{1}(\Omega)=0=\mathcal{I}_{2}(\Omega) .
$$

If the function $v$ is strictly increasing, then the previous and (A.11) easily imply that $|\Omega \Delta K|=0$, i.e. $\Omega$ has to coincide with $K$. On the contrary, if $v$ is simply a non decreasing function, the proof is a bit more complicated. In this case, the information $\mathcal{I}_{2}(\Omega)=0$ is useless and we need to exploit the first one i.e. $\mathcal{I}_{1}(\Omega)=0$. Keeping into account that $V(t)>0$ for $t>0$, from the latter we can infer that

$$
\left\|\nu_{\Omega}(x)\right\|_{*}=\left\langle\frac{x}{\|x\|}, \nu_{\Omega}(x)\right\rangle, \quad \text { for } \mathcal{H}^{N-1} \text {-a.e. } x \in \partial \Omega .
$$

This implies that the standard anisotropic perimeter of $\Omega$ can be written as

$$
P_{K}(\Omega)=\int_{\partial \Omega}\left\|\nu_{\Omega}(x)\right\|_{*} d \mathcal{H}^{N-1}(x)=\int_{\Omega} \operatorname{div}\left(\frac{x}{\|x\|}\right) d x=\int_{\Omega} \frac{N-1}{\|x\|} d x,
$$

where we used the computations of Lemma A.3, with $V \equiv 1$. We now observe that the last integrand is a strictly decreasing function of $\|\cdot\|$. Then using that $K=\{x:\|x\| \leq 1\}$ and that $|\Omega|=|K|$, we have

$$
\begin{aligned}
\int_{\Omega} \frac{N-1}{\|x\|} d x & \leq \int_{\Omega \cap K} \frac{N-1}{\|x\|} d x+(N-1)|\Omega \backslash K| \\
& =\int_{\Omega \cap K} \frac{N-1}{\|x\|} d x+(N-1)|K \backslash \Omega| \leq \int_{K} \frac{N-1}{\|x\|} d x
\end{aligned}
$$

with strict inequality if $|\Omega \Delta K| \neq 0$. This implies that $P_{K}(\Omega) \leq P_{K}(K)$ and $P_{K}(\Omega)<P_{K}(K)$ as soon as $|\Omega \Delta K| \neq 0$. By appealing to the equality cases in the Wulff inequality (A.2), we then conclude that $|\Omega \Delta K|=0$, that is $\Omega$ coincides with $K$ also in this case.

Remark A.5. As in the isotropic case considered in [7], here as well one can prove a sharp quantitative version of the weighted Wulff inequality (A.8). See [17, Theorem 7.4.1] for more details. 
Some significant instances of functions $V$ satisfying our hypotheses (A.3) and (A.4) are given by convex powers, i.e.

$$
V(t)=t^{\beta}, \quad t \geq 0,
$$

for every $\beta \geq 1$. In particular, by choosing as $K$ the unit ball $B_{p}$ of the $\ell^{p}$ norm centered at the origin, i.e.

$$
B_{p}=\left\{x \in \mathbb{R}^{N}:\|x\|_{\ell^{p}}<1\right\}
$$

and by using the distinguished notation

$$
P_{p, \beta}(\Omega)=\int_{\partial \Omega}\|x\|_{\ell^{p}}^{\beta}\left\|\nu_{\Omega}(x)\right\|_{\ell^{p^{\prime}}} d \mathcal{H}^{N-1}(x),
$$

we have the following particular case of Theorem A.4, that we enunciate as a separate result.

Corollary A.6. Let $p \geq 1$ and $\beta \geq 1$, for every $\Omega \subset \mathbb{R}^{N}$ open bounded Lipschitz set, we have

$$
P_{p, \beta}(\Omega) \geq N\left|B_{p}\right|^{\frac{1-\beta}{N}}|\Omega|^{\frac{N+\beta-1}{N}},
$$

with equality if and only if $\Omega$ coincides with $B_{p}$, up to dilations.

\section{References}

[1] Acerbi, E., Fusco, N.: Regularity of minimizers of non-quadratic functionals: the case $1<p<2$. J. Math. Anal. Appl. 140, 115-135 (1989)

[2] Alessandrini, G., Magnanini, R.: Elliptic equations in divergence form, geometric critical points of solutions, and Stekloff eigenfunctions. SIAM J. Math. Anal. 25, 1259-1268 (1994)

[3] Beauzamy, B.: Introduction to Banach Spaces and Their Geometry. NorthHolland Mathematics Studies, vol. 68. North-Holland Publishing Co., Amsterdam (1985)

[4] Belloni, M., Kawohl, B.: The pseudo $p$-Laplace eigenvalue problem and viscosity solution as $p \rightarrow \infty$. ESAIM Control Optim. Calc. Var. 10, 28-52 (2004)

[5] Belloni, M., Kawohl, B.: A direct uniqueness proof for equations involving the p-Laplace operator. Manuscr. Math. 109, 229-231 (2002)

[6] Betta, M.F., Brock, F., Mercaldo, A., Posteraro, M.R.: A weighted isoperimetric inequality and applications to symmetrization. J. Inequal. Appl. 4, 215-240 (1999)

[7] Brasco, L., De Philippis, G., Ruffini, B.: Spectral optimization for the Stekloff-Laplacian: the stability issue. J. Funct. Anal. 262, 4675-4710 (2012)

[8] Brasco, L., Franzina, G.: A note on positive eigenfunctions and hidden convexity. Arch. Mat. 99, 367-374 (2012) 
[9] Brock, F.: An isoperimetric inequality for eigenvalues of the Stekloff problem. ZAMM Z. Angew. Math. Mech. 81, 69-71 (2001)

[10] Cuesta, M.: Minimax theorems on $C^{1}$ manifolds via Ekeland variational principle. Abstr. Appl. Anal. 13, 757-768 (2003)

[11] Cuesta, M., De Figueiredo, D.G., Gossez, J.-P.: The beginning of the Fucik spectrum for the $p$-Laplacian. J. Differ. Equ. 159, 212-238 (1999)

[12] Cuesta, M., De Figueiredo, D.G., Gossez, J.-P.: A nodal domain property for the $p$-Laplacian. C. R. Acad. Sci. Paris 330, 669-673 (2000)

[13] Dacorogna, B., Gangbo, W., Subía, N.: Sur une généralisation de l'inégalité de Wirtinger. Ann. Inst. H. Poincaré Anal. Non Linéaire 9, 29-50 (1992)

[14] DiBenedetto, E.: $C^{1+\alpha}$ local regularity of weak solutions of degenerate elliptic equations. Nonlinear Anal. 7, 827-850 (1983)

[15] Drábek, P., Robinson, S.B.: Resonance problems for the $p$-Laplacian. J. Funct. Anal. 169, 189-200 (1999)

[16] Figalli, A., Maggi, F., Pratelli, A.: A mass transportation approach to quantitative isoperimetric inequalities. Invent. Math. 81, 167-211 (2010)

[17] Franzina, G.: Existence, uniqueness, optimization and stability for low eigenvalues of some nonlinear operators. $\mathrm{PhD}$ thesis, Università di Trento. http:// cvgmt.sns.it/paper/2102/ (2012)

[18] Franzina, G., Lamberti, P.D.: Existence and uniqueness for a $p$-Laplacian nonlinear eigenvalue problem. Electron. J. Differ. Equ. 26, 1-10 (2010)

[19] Fučík, S., John, O., Nečas, J.: On the existence of Schauer bases in Sobolev spaces. Comment. Math. Univ. Carol. 13, 163-175 (1972)

[20] Fučík, S., Nečas, J., Souček, J., Souček, V.: Spectral analysis of nonlinear operators. Lecture Notes in Mathematics, vol. 346. Springer, Berlin (1973)

[21] García Azorero, J.P., Peral Alonso, I.: Existence and nonuniqueness for the p-Laplacian. Commun. Part. Differ. Equ. 12, 1389-1430 (1987)

[22] Giusti, E.: Direct Methods in the Calculus of Variations. World Scientific Publishing, Singapore (2003)

[23] Henrot, A.: Extremum Problems for Eigenvalues of Elliptic Operators. Frontiers in Mathematics. Birkhäuser, Basel (2006)

[24] Juutinen, P., Lindqvist, P.: On the higher eigenvalues for $\infty$-eigenvalue problem. Calc. Var. Partial Differ. Equ. 23, 169-192 (2005)

[25] Kuttler, J.R., Sigillito, V.G.: An inequality for a Stekloff eigenvalue by the method of defect. Proc. Am. Math. Soc. 20, 357-360 (1969)

[26] Lieberman, G.M.: Boundary regularity for solutions of degenerate elliptic equations. Nonlinear Anal. 12, 1203-1219 (1988) 
[27] Lindqvist, P.: Notes on the p-Laplace equation. Report. University of Jyväskylä Department of Mathematics and Statistics, 102. University of Jyväskylä, Jyväskylä (2006)

[28] Matveev, O.V.: Bases in Sobolev spaces on bounded domains with Lipschitz boundary. Mat. Zametki 72, 408-417 (2002)

[29] Schneider, R.: Convex Bodies: the Brunn-Minkowski Theory. Cambridge University Press, Cambridge (1993)

[30] Struwe, M.: Variational methods. Applications to nonlinear partial differential equations and Hamiltonian systems, 4th edn. Ergebnisse der Mathematik und ihrer Grenzgebiete. 3. Folge. A Series of Modern Surveys in Mathematics, vol. 34. Springer, Berlin (2008)

[31] Takáč, P., Tello, L., Ulm, M.: Variational problems with a $p$-homogeneous energy. Positivity 6, 75-94 (2002)

[32] Uralt'seva, N., Urdaletova, N.: The boundedness of the gradients of generalized solutions of degenerate quasilinear nonuniformly elliptic equations. Vest. Leningr. Univ. Math. 16, 263-270 (1984)

[33] Weinstock, R.: Inequalities for a classical eigenvalue problem. J. Ration. Mech. Anal. 3, 745-753 (1954)

Lorenzo Brasco

Laboratoire d'Analyse

Topologie

Probabilités

Aix-Marseille Université

39 Rue Frédéric Joliot Curie

13453 Marseille Cedex 13

France

e-mail: lorenzo.brasco@univ-amu.fr

Giovanni Franzina

Department Mathematik

Universität Erlangen-Nürnberg

Cauerstraße 11

91058 Erlangen Germany

e-mail: franzina@math.fau.de

Received: 10 October 2012.

Accepted: 16 March 2013. 